\title{
Escaping the evolutionary trap: can size-related contest advantage compensate for juvenile mortality disadvantage when parasitoids develop in unnatural invasive hosts?
}

\author{
Mike Mesterton-Gibbons \\ Department of Mathematics, Florida State University, 1017 Academic Way, Tallahassee, Florida 32306-4510, U.S.A \\ Antonino Cusumano \\ Department of Agricultural, Food And Forest Sciences (SAAF), University of Palermo, Viale delle Scienze, 90128 \\ Palermo, Italy \\ Ian C.W. Hardy \\ School of Biosciences, University of Nottingham, Sutton Bonington Campus, Loughborough, Leics LE12 5RD, U.K.
}

\begin{abstract}
The quality of hosts for a parasitoid wasp may be influenced by attributes such as host size or species, with high quality for successful development usually coincident with high quality for larger offspring. This is not always the case: for the Scelionid wasp Trissolcus basalis, oviposition in eggs of the Brown Marmorated Stink Bug, Halyomorpha halys, rather than of the normal host, the Southern Green Stink Bug, Nezara viridula, leads to lower offspring survival, but survivors can be unusually large. Adult female T. basalis engage in contests for host access. As larger contestants are typically favoured in contests between parasitoids, the larger size of surviving offspring may compensate for the mortality of others. We construct a general game-theoretic model to explore whether size advantage can sustain a maternal preference to utilize a more deadly host species. We find that size advantage alone is unlikely to sustain a shift in host preference, yet such an outcome is possible when size asymmetries act simultaneously with advantages in host possession (ownership effect). H. halys is an invasive pest of major agro-economic importance in Europe and the Americas, and use of its eggs as hosts by native parasitoids such as $T$. basalis has been seen as an evolutionary trap due to high developmental mortality. Our model suggests that the recently discovered effect of host choice on offspring size may provide an escape from the trap via effects on contest biology of T. basalis which could
\end{abstract}

Email addresses: mesterto@math.fsu.edu (Mike Mesterton-Gibbons), antonino.cusumano@unipa.it (Antonino Cusumano), ian.hardy@nottingham.ac.uk (Ian C.W. Hardy) 
foster a more stable association with $H$. halys. An evolutionary shift in the reproductive value of $H$. halys could increase the efficiency of $T$. basalis as a biological control agent of this invasive stink bug pest.

Keywords: Host species, Reproductive value, Size advantage, Ownership advantage, Evolutionarily stable strategy, Trissolcus basalis, Brown Marmorated Stink Bug, Halyomorpha hyalis 


\section{Introduction}

Juvenile parasitoid wasps develop on the resources provided by the body of a single host. The quality and quantity of the resource can be influenced by, for instance, the host's size and by its developmental stage and these attributes may then be manifest in terms of parasitoid developmental mortality and/or the size, fecundity and longevity of surviving offspring, all of which are components of evolutionary fitness (Godfray, 1994). Many species of parasitoids can develop from several or even many species of hosts (oligophagy and polyphagy, respectively) and variation, in terms of nutritional composition, size and defences against parasitism, between host species can be a major determinant of parasitoid fitness parameters, in turn influencing host acceptance decisions by foraging adult females.

In some parasitoids, development in a given host species may negatively influence the probability of offspring survival to adulthood and yet positively influence the characteristics of those offspring that do survive. This is the case in Trissolcus basalis (Hymenoptera: Sceleonidae): oviposition into eggs of the invasive Brown Marmorated Stink Bug (Halyomorpha halys, Hemiptera: Pentatomidae) leads to far lower offspring survival (with estimates ranging from $0-6 \%$ (Rondoni et al., 2017; Peri et al., 2020) to 38\% Balusu et al. (2019) than oviposition into eggs of its main host, the Southern Green Stink Bug (Nezara viridula, Hemiptera: Pentatomidae) (Cusumano et al., 2011; Peri et al., 2020), but female offspring that survive are typically much larger (25\% increase in tibia length, Peri et al., 2020). Among adult female parasitoid wasps, larger size is generally associated with higher fecundity (Hardy et al., 1992) and foraging ability (Karsai et al., 2006; Visser, 1994). One aspect of foraging is the ability to competitively acquire and subsequently defend hosts or patches of hosts against other foraging females, and female T. basalis engage in such contests (Field \& Calbert, 1998). Across the parasitoid Hymenoptera, larger contestants are typically favoured in agonistic interactions between adults (Hardy et al., 2013) and such body size effects can influence reproductive decisions by foraging females in a game-theoretic manner (e.g. clutch size optima, (Mesterton-Gibbons \& Hardy, 2004; Goubault et al., 2007).

Here we develop a game-theoretic model to address the following general question: Can size advantage in contests among adults sustain a preference for a more deadly (in terms of offspring developmental mortality) host by foraging females? For greatest generality, and with future work in mind, we first formulate the model (in $\S 2$ and especially Appendix A) in terms of a five-dimensional parameter space. The parameters are: 1 . The reproductive value of the more deadly host relative to that of the natural host, $\alpha ; 2$. The 
proportion of hosts that are of the more deadly species, $\theta ; 3$. The probability that a host is never found, $k$; The probability a large wasp outcompetes a normal wasp relative to the probability a normal wasp outcompetes a large wasp, $\lambda$; and 5 . The owner advantage, increase in probability beyond 0.5 of owner winning contest with intruder of equal size, $\rho$. The model thus integrates non-contest $(\alpha, \theta, k)$ and contest-related $(\lambda, \rho)$ considerations. All five parameters are dimensionless and in principle measurable, but in practice their measurement has yet to be addressed by empirical studies. As clarity of prediction in theoretical work decreases with the numbers of parameters considered, we then focus ( $(3)$ on the subset of the parameter space where size advantage in contests is most relevant, thus reducing the dimension of the parameter space from five to two. We subsequently revisit the higher-dimensional parameter space ( $(4)$ and in our concluding discussion ( $(5)$.

Our model has a wide set of potential applications, given that species invasions are likely to occur increasingly frequently due to both international transport and global climate change, and that invasive species will be encountered by resident natural enemies with which they have not co-evolved (Berthon, 2015; Abram et al., 2017), leading to a range of possible empirical values of the parameters we consider. The model is nonetheless of most immediate use in considering the invasion of European and American cropping systems by the Brown Marmorated Stink Bug (Rice et al., 2014; Leskey \& Nielsen, 2018) as the use of its eggs as hosts by native parasitoids such as T. basalis has been seen as an evolutionary trap due to the high developmental mortality of offspring (Abram et al., 2014; Costi et al., 2020). However, the recently discovered effects of host species on offspring size may provide an escape from the trap by providing a fitness advantage to surviving offspring via enhanced performance in contests for future hosts.

\section{Mathematical model}

For the sake of simplicity, we consider a population of female-producing female parasitoids (thelytoky). Likewise for simplicity, we assume that there are only two adult body sizes, large and normal, and that each egg surviving on a more deadly (Halyomorpha hyalis-like) host becomes a large adult, whereas each egg surviving on a natural host becomes a normal adult.

This population consists of three different types or strategies, distinguished by the type of host they are willing to exploit. A $C$-strategist is the customary obligate exploiter of natural hosts, so its progeny are always of normal size. A $D$-strategist is an obligate exploiter of the more deadly host (e.g., H. hyalis), so its progeny are always large. A $U$-strategist is an undiscerning exploiter: its progeny are normal or large according to whether it happens to have exploited a natural host or a more deadly one. Let these three 
types occur in proportions $x_{1}, x_{2}$ and $x_{3}$, respectively, so that $C$ is strategy $1, D$ is strategy 2 and $U$ is strategy 3.

As in Mesterton-Gibbons \& Hardy (2004), we assume that an animal's reward is the expected number of surviving offspring from a suitable host. Without loss of generality, let the value of a natural host in terms of the average number of surviving offsprint from it be defined as the unit of fitness, and let the corresponding value of a more deadly host be $\alpha$ units, where $\alpha<1$ because the parasitoid has much higher developmental mortality on the more deadly host $(94 \%)$ compared with levels observed on the natural host (16\%) (Peri et al., 2020). Note that $\alpha$ represents parasitoid survival and fecundity on a more deadly host relative to that on a natural host, with "natural" developmental mortality and fecundity built into the unit of fitness by our definition. For example, with $95 \%$ mortality on a more deadly host compared to $20 \%$ on natural hosts but with the same fecundity we would have $\alpha=0.05 / 0.8=0.0625$; whereas if in addition survivors from more deadly hosts were three times as fecund as natural survivors we would have $\alpha=3 \times 0.05 / 0.8=0.1875$.

We assume that the frequencies of more deadly and natural hosts are $\theta$ and $1-\theta$, respectively, and hence that more deadly and natural hosts are located at rates proportional to $\theta$ and $1-\theta$. Correspondingly, in the absence of differential survival and fecundity, progeny of a $U$-strategist would be $\frac{\theta}{1-\theta}$ times as likely to be large as to be normal. However, an egg is only $\alpha$ times as likely to survive and reproduce on a more deadly host as on a natural one. Hence progeny of a $U$-strategist are only $\alpha \cdot \frac{\theta}{1-\theta}$ times as likely to be large as to be normal. So the probability that a randomly selected $U$-strategist is large or normal is $\omega_{L}$ or $\omega_{N}$, respectively, where

$$
\omega_{L}=\frac{\alpha \theta}{\alpha \theta+1-\theta}, \quad \omega_{N}=\frac{1-\theta}{\alpha \theta+1-\theta} .
$$

Note that we assume

$$
0<\alpha, \theta<1
$$

throughout, so that (1) implies $0<\omega_{L}, \omega_{N}<1$ as well.

We assume that, in contests between two large or between two normal individuals, where neither has any advantage in terms of size, it is possible that prior ownership may confer an advantage instead. Specifically, in a contest between two individuals of equal size, the owner wins with probability $q_{O}$ and the intruder wins with probabilty $q_{I}$, where

$$
q_{O}=\frac{1}{2}(1+\rho), \quad q_{I}=\frac{1}{2}(1-\rho)
$$

with $0 \leq \rho \leq 1$. We refer to $\rho$ as owner advantage: it measures the extent beyond 
equiprobability to which an owner is favoured to win in the event of a fight between equals.

In contests between large and normal individuals, however, greater size does confer an advantage on the larger individual. Let a large individual be $\lambda$ times more likely to win against an individual of normal size than it would if it were itself of normal size, where $\lambda>1$. Then in a contest between a large owner and an intruder of normal size, the owner wins with probability $q_{O L}$ and the intruder wins with probability $q_{I N}$, where

$$
q_{O L}=\frac{\lambda(1+\rho)}{1-\rho+\lambda(1+\rho)}, \quad q_{I N}=\frac{1-\rho}{1-\rho+\lambda(1+\rho)} .
$$

Likewise, in a contest between a normal owner and a large intruder, the owner wins with probability $q_{O N}$ and the intruder wins with probability $q_{I L}$, where

$$
q_{O N}=\frac{1+\rho}{1+\rho+\lambda(1-\rho)}, \quad q_{I L}=\frac{\lambda(1-\rho)}{1+\rho+\lambda(1-\rho)} .
$$

As in Mesterton-Gibbons \& Hardy (2004), we assume that there is a narrow timewindow in which a parasitoid can actually acquire a host and thus that each host is the subject of at most one contest. To be able to reproduce, females must either find a suitable unguarded host and defend it against at most one intruder or take over a suitable guarded host in a contest.

Let $Z(T)$ be the probability that a host is located by time $T$; let $Y(T)$ be the probability that a host is located some time after time $T$; and let $k$ be the probability that a host is never found (during the entire vulnerable period of its development). Then multiplying the probability $1-Z(T)$ that a host has not been found at time $T$ by the probability $1-Y(T)$ that it is not subsequently found yields

$$
(1-Z(T))(1-Y(T))=k
$$

for any $T$ : the bigger the value of $Z(T)$, the smaller the value of $Y(T)$. We assume that time to next arrival at the host follows an exponential distribution with parameter $a$, so that $Z(T)=1-e^{-a T}, Y(T)=1-e^{-a(p-T)}$ and

$$
k=e^{-a T} e^{-a(p-T)}=e^{-a p}
$$

by (6), where $p$ denotes the length of the vulnerable period of development for a host. We further assume that location time $T$ for a focal individual is uniformly distributed between 0 and $p$, so that the average probability of a host being unguarded is

$$
\gamma=\mathrm{E}[1-Z(T)]=1-\int_{0}^{p} \frac{1}{p} Z(t) d t=\frac{k-1}{\ln (k)}
$$


where E denotes expected value.

We can now proceed to calculate expressions for the fitnesses to each of the three strategies, which appear in Appendix A. These expressions depend explicitly on $\alpha, \theta, \rho$, $\lambda$ and $k$ (both directly and indirectly through $\gamma$ ), but do not depend directly on either $a$ or $p$, whose effect on fitness is subsumed by $k$ through (7). We therefore regard $\alpha, \theta, \rho, \lambda$ and $k$ as the five dimensionless parameters of the model. The corresponding evolution of the strategy frequencies is governed by the replicator equations (Taylor \& Jonker, 1978; Hofbauer \& Sigmund, 1998). For $i=1,2,3$, let $W_{i}$ denote the fitness to strategy $i$, whose frequency is $x_{i}$. Then the governing equations are

$$
\frac{d x_{i}}{d t}=x_{i}\left\{W_{i}-\bar{W}\right\}, \quad i=1, \ldots, 3,
$$

where $t$ denotes time and

$$
\bar{W}=\sum_{i=1}^{3} x_{i} W_{i}
$$

is the average fitness of the entire population. Note that, because

$$
x_{1}+x_{2}+x_{3}=1,
$$

the first two equations in (9) imply the third, which therefore we do not need for the analysis that follows.

\section{Analysis of the reduced model}

The expressions for fitness calculated in Appendix A in general depend on the strategy frequencies $x_{1}, x_{2}, x_{3}$ and on the five dimensionless parameters $\alpha, \theta, \rho, \lambda$ and $k$, and in general are too complex for tractable analysis. Therefore, we focus our attention on the important limit as $a \rightarrow 0$ in (7), in which all hosts are eventually found, and thus contest behaviour is most relevant. In this limit, which can be regarded as the limit of high parasitoid density, the expressions for fitness simplify significantly (Appendix B). Because the correct expressions for fitness are obtained by setting $k=0$ and hence $\gamma=0$ in (A.6), (A.9) and (A.17), we will refer to this limit largely as the $k=0$ limit (as opposed to the $a \rightarrow \infty$ limit). To make things as simple as possible, however, we now also assume that there is no owner advantage and that greater size is always decisive in a contest, in the sense that a large individual always wins agains a normally sized one. Thus $\rho=0$, $\lambda \rightarrow \infty$ in addition to $k=0$, and (3)-(5) reduce to

$$
q_{0}=q_{I}=\frac{1}{2}, \quad q_{O L}=q_{I L}=1, \quad q_{O N}=q_{I N}=0 .
$$


We refer to this model as our reduced model. The evolution of the population now depends only on two dimensionless parameters, namely, the relative value $\alpha$ and frequency $\theta$ of the more deadly host, and on the proportions $x_{1}, x_{2}$ of $C$ and $D$ strategists, with the proportion $x_{3}$ of $U$ implied by (11).

In a theoretical exploration, focusing on a low-dimensional parameter space facilitates clarity of prediction. Nevertheless, departures from the above assumptions are briefly discussed in $\S 4$, where we consider the effects of different values of the other three parameters from the values assumed here (specifically, in $\S 4$ we consider finite $\lambda$ and positive $\rho$ and $k$ ).

In general, what we wish to know is the strategy mix to which the population ultimately evolves, which now depends only on $\alpha$ and $\theta$ as noted above. Thus, for any point $(\alpha, \theta)$ inside the parameter square

$$
S=\{(\alpha, \theta) \mid 0 \leq \alpha, \theta \leq 1\}
$$

in Figure 1 we wish to know the point or set of points in the phase-plane triangle

$$
\Delta=\left\{\left(x_{1}, x_{2}\right) \mid 0 \leq x_{1}+x_{2} \leq 1\right\}
$$

in Figure 2 to which the vector $\left(x_{1}(t), x_{2}(t)\right)$ of proportions of $C$ - and $D$ - strategists ultimately evolves. This final destination is typically a static equilibrium point, in which case we may denote it by $\left(x_{1}(\infty), x_{2}(\infty)\right)$; but for a small subregion of $S$ the final destination is instead a dynamic equilibrium or limit cycle, as we shall see in due course.

Equations (9)-(12) and (B.1)-(B.3) imply that the evolution of $\left(x_{1}(t), x_{2}(t)\right)$ towards its final destination is governed by

$$
\frac{d x_{1}}{d t}=x_{1} \phi_{1}\left(x_{1}, x_{2}\right), \quad \frac{d x_{2}}{d t}=x_{2} \phi_{2}\left(x_{1}, x_{2}\right)
$$

with

$$
\begin{aligned}
\phi_{1}\left(x_{1}, x_{2}\right)= & \frac{1}{2}\left\{(1-\theta) x_{2}^{3}-\left(1-x_{1}\right)\left\{\theta\left(\alpha\left\{1+x_{1}^{2}\right\}-1-\omega_{L} x_{1}\right)+\omega_{L}\right\}\right. \\
& +x_{2}{ }^{2}\left\{\alpha(1-\theta)\left(x_{1}+1\right)\left(1-\omega_{L}\right)-\theta\left(1+\omega_{L}\right) x_{1}+\theta+\omega_{L}\right\} \\
& \left.\quad+x_{2}\left\{\alpha(1-\theta)\left\{\left(1-x_{1}^{2}\right) \omega_{L}-2\right\}-\theta\left(1+\omega_{L} x_{1}^{2}\right)+1+\omega_{L} x_{1}\right\}\right\}
\end{aligned}
$$

and

$$
\begin{array}{r}
\phi_{2}\left(x_{1}, x_{2}\right)=\frac{1}{2}\left\{\alpha\left(1-x_{2}\right)\left(1+x_{1}^{2}\right)-x_{1}\left\{x_{1}\left(1+x_{2}\right)+2 x_{2}^{2}\right\}\right\} \\
+\left(1-x_{1}-x_{2}\right)\left\{\frac{1}{2}\left\{\alpha\left(1-x_{2}\right)\left(1+x_{1}\right)-x_{1}\left(1+x_{2}\right)\right\} \omega_{N}-\alpha \theta K\left(x_{1}, x_{2}, \omega_{L}\right)\right. \\
\left.-(1-\theta) K\left(x_{2}, x_{1}, \omega_{N}\right)-(1-\theta)\left(1+x_{2}\right) \omega_{L} x_{1}\right\}
\end{array}
$$



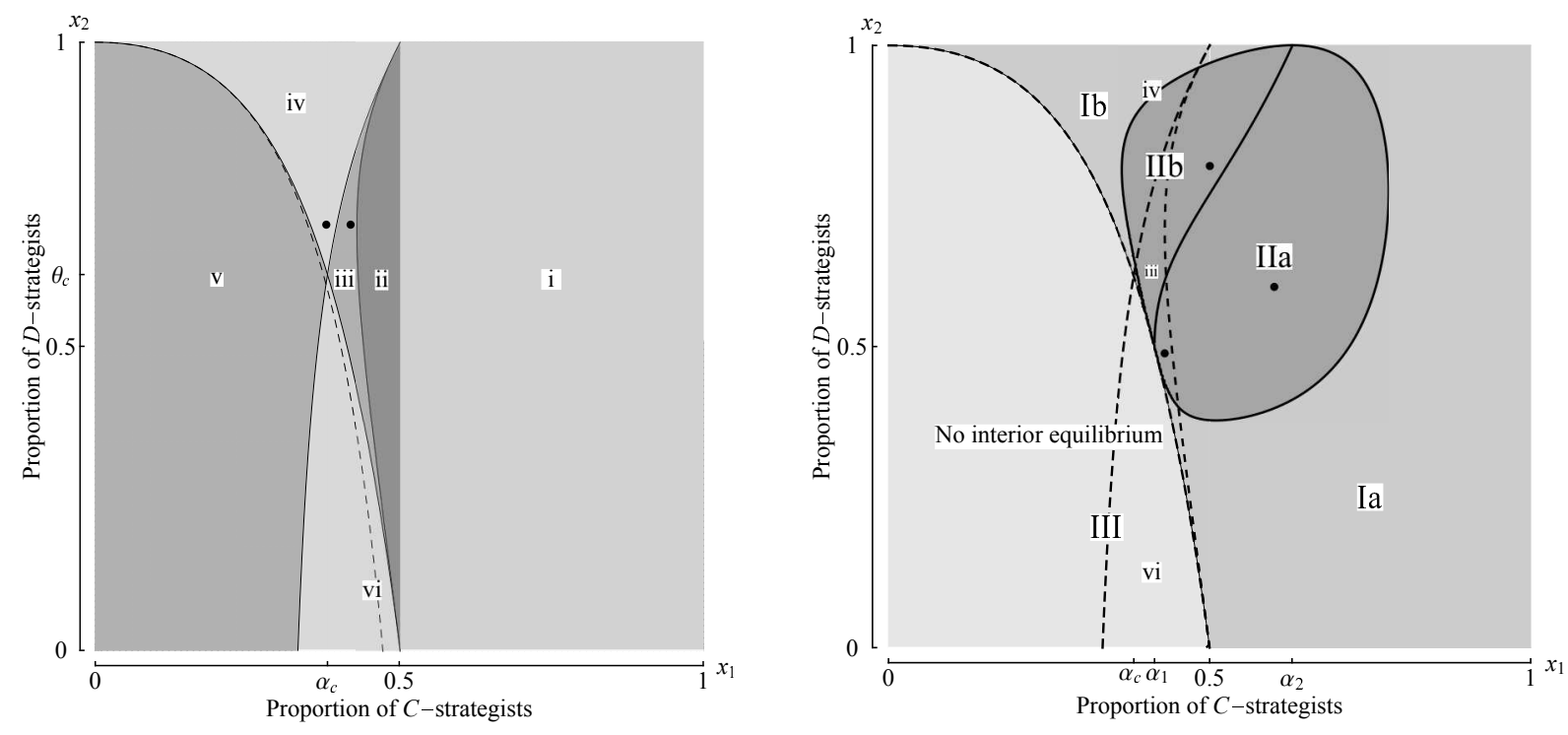

Figure 1: The parameter square $S$.

(a) Regions corresponding to equilibria on the boundary of $\Delta$. The curve from $\left(\frac{1}{2}, 1\right)$ to $\left(\frac{1}{2}, 0\right)$ between regions ii and iii has equation $4 \theta^{2} \alpha^{3}+12 \theta(1-\theta) \alpha^{2}+\left(11 \theta^{2}-20 \theta+8\right) \alpha=4(1-\theta)^{2}$. The curve from $(0,1)$ to $\left(\frac{1}{2}, 0\right)$ between regions $\mathrm{v} \cup$ vi and iii $\cup$ iv has equation $\theta=(1-2 \alpha) /(1-\alpha)^{2}$. The curve from $\left(\frac{1}{2}, 1\right)$ to $\left(\frac{1}{3}, 0\right)$ between regions iv $\cup \mathrm{v}$ and iii $\cup$ vi has equation $\theta=(1-3 \alpha) /\left\{2(1-\alpha)^{2}-1\right\}$. These curves cross at $\left(\alpha_{c}, \theta_{c}\right)$, where $\alpha_{c}=\frac{1}{2}(3-\sqrt{5}) \approx 0.382$ and $\theta_{c}=\frac{1}{2}(\sqrt{5}-1) \approx 0.618$. The dots at $(\alpha, \theta)=(0.38,0.7)$ and $(0.42,0.7)$ correspond to Figure 2 ; the dashed curve joining $(0,1)$ to $2(\sqrt{5}-2), 0) \approx(0.472,0)$ and other details are discussed in Appendix D. The following table excludes a saddle point at $(0,0)$ and an unstable node at $(0,1)$, both of which always exist.

i Unstable nodes at $(1,0)$ and saddle points at $\left(0, x_{2 s}\right)$ and $\left(x_{1 r}, x_{2 r}\right)$, where $x_{1 r}+x_{2 r}=1$

ii Saddle points at $(1,0)$ and $\left(0, x_{2 s}\right)$

iii A stable node at $\left(x_{1 b}^{+}, 0\right)$ and saddle points at $\left(x_{1 b}^{-}, 0\right),\left(0, x_{2 s}\right)$ and $(1,0)$

iv A stable node at $(1,0)$ and saddle points at $\left(x_{1 b}^{+}, 0\right)$ and $\left(0, x_{2 s}\right)$

$\mathrm{v} \quad$ A stable node at $(1,0)$

vi A stable node at $\left(x_{1 b}^{+}, 0\right)$

(b) Regions corresponding to a (static) equilibrium in the interior of $\Delta$. The curve between regions IIa and IIb joins $\left(\alpha_{1}, \frac{1}{2}\right)$ to $\left(\alpha_{2}, 1\right)$ where $\alpha_{1}=\sqrt{2}-1 \approx 0.414$ and $\alpha_{2} \approx 0.628$. The dots at $(\alpha, \theta)=(0.6,0.6)$, $(0.43,0.49)$ and $(0.5,0.8)$ correspond to Figures 3 and 4 . For further details see Appendix D.

Ia A stable node at $\left(p_{*}, p_{*}\right)$

$\mathrm{Ib} \quad$ An unstable node at $\left(p_{*}, p_{*}\right)$

IIa A stable focus at $\left(p_{*}, p_{*}\right)$

IIb An unstable focus at $\left(p_{*}, p_{*}\right)$

III No interior equilibrium point 
(a) $\alpha=0.38, \theta=0.7, \rho=0, \lambda \rightarrow \infty, k=0$

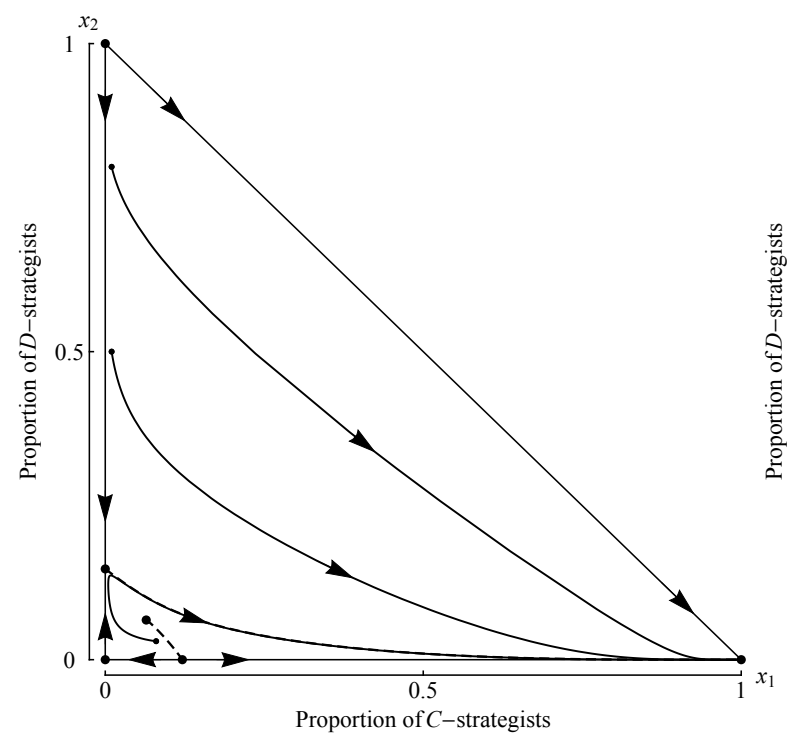

(b) $\alpha=0.42, \theta=0.7, \rho=0, \lambda \rightarrow \infty, k=0$

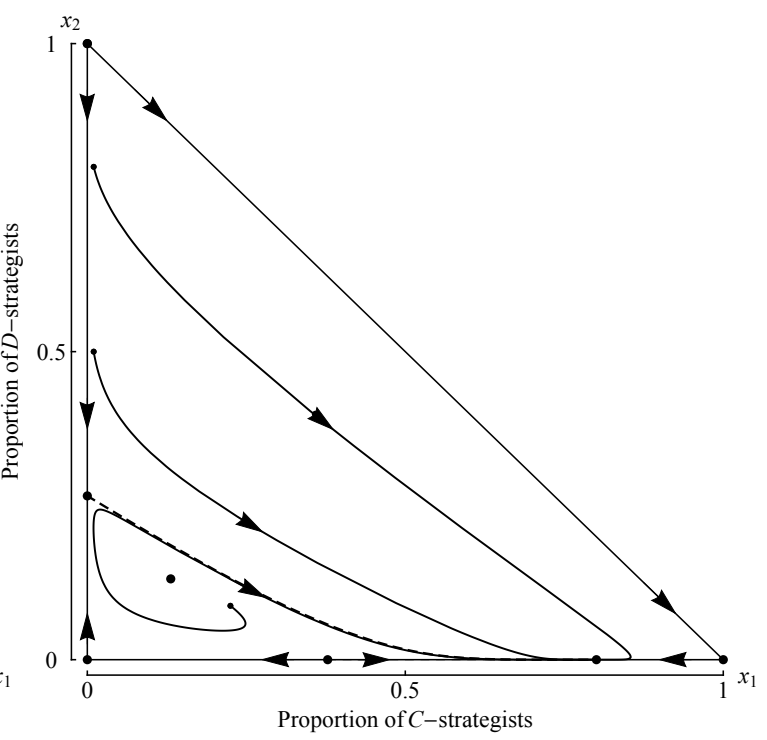

Figure 2: Phase-plane triangles $\Delta$ corresponding to two different points in regions iii and iv of Figure 1(a). Large dots denote equilibrium points, small dots denote points at which sample trajectories begin. (a) This phase-plane triangle corresponds to the dot in region iv of Figure 1(a). There are 6 equilibrium points: saddle points at $(0,0),(0,0.1473)$ and $(0.1212,0)$, an unstable node at $(0,1)$, an unstable focus at $(0.0644,0.0644)$ and a stable node at $(1,0)$. The vertex $(1,0)$ is the only local attractor and therefore also the global attractor: the population evolves to a monomorphism of $C$. Three sample trajectories are shown, with initial points $(0.01,0.8),(0.01,0.5)$ and $(0.08,0.03)$. (b) This phase-plane triangle corresponds to the dot in region iii of Figure 1(a). There are now 7 equilibrium points: saddle points at $(0,0),(0,0.2659)(0.3779,0)$ and $(1,0)$, an unstable node at $(0,1)$, an unstable focus at $(0.1312,0.1312)$ and a stable node at $(0.8005,0)$. This base-edge equilibrium is the only local attractor and therefore also the global attractor: the population evolves to a polymorphism of $C$ and $U$. Three sample trajectories are shown again; two initial points are the same, the third has been changed from $(0.225,0.0875)$ to $(0.01,0.8)$. Separatrices are shown dashed. In both cases, a separatrix joins the unstable focus to a base-edge saddle point and the side-edge saddle point to the global attractor, although in (a) this separatrix is scarcely visible behind one of the trajectories. 
where we have defined

$$
K(p, q, \omega)=p^{2}+\frac{1}{2}(1+p)(1-p-q+\omega q)
$$

for any size probability $\omega$ and proportions $p, q$. In view of (1), the right-hand sides of (15) depend only on the proportions $x_{1}, x_{2}$ of strategies $C, D$ and on the two dimensionless parameters $\alpha$ and $\theta$. The evolution of $\left(x_{1}(t), x_{2}(t)\right)$ over the triangle $\Delta$ can now be determined from (16) by standard methods of nonlinear analysis (e.g., Strogatz, 2014; Layek, 2015), with the proportion of strategy $U$ simultaneously determined from (11) as $x_{3}(t)=1-x_{1}(t)-x_{2}(t)$.

We begin by noting that size advantage, although decisive, would have no effect in the complete absence of $U$-strategists because in that case all contests would occur between equals. Rather, $C$ would go to fixation when its fitness exceeded that of a $D$-strategist by twice or more, thus completely offsetting the $50 \%$ chance of losing a contest, that is, when $\alpha<\frac{1}{2}$; whereas both strategies would persist when $\frac{1}{2}<\alpha<1$, with the proportion of $D$ in the strategy mix increasing from 0 to $\frac{1}{2}$ as $\alpha$ increased from $\frac{1}{2}$ to 1 . This intuition is readily confirmed by analysis; see Appendix $C$, where an explicit expression is given for the proportions of $C$ and $D$ when both persist. However, this no- $U$ equilibrium, which occurs on the "roof-edge" of $\Delta$ where $x_{1}+x_{2}=1$, fails to persist as soon as a single $U$-strategist enters the population: in $\Delta$ it is an unstable saddle point, which can be approached only along the line where $x_{3}=0$. (This saddle point does not appear in Figure 2 because the phase plane is sketched for $\alpha<\frac{1}{2}$ in both panels, but it does appear in Figure 3(a) where $\alpha>\frac{1}{2}$.)

We now proceed with a summary of the more general analysis, whose details are presented in appendices. Inspection of (16) reveals that all three vertices of $\Delta$ are invariably equilibrium points because $\phi_{1}(1,0)=\phi_{2}(0,1)=0$; however, $(0,0)$ and $(0,1)$ are both unstable, $(0,0)$ being a saddle point and $(0,1)$ an unstable node (Appendix D). By contrast, the type of $(1,0)$ depends on where $(\alpha, \theta)$ lies in $S$ : from Appendix D, it is an unstable node, a saddle point or a stable node according to whether $(\alpha, \theta)$ lies in region i, region ii $\cup$ iii $\cup$ vi or region iv $\cup v$ of Figure 1 (a). These points are illustrated by Figure 2, where $(1,0)$ is a stable node in (a) but a saddle point in (b), whereas $(0,0)$ is a saddle point and $(0,1)$ is an unstable focus in both panels.

Depending on the values of $\alpha$ and $\theta$, up to four additional types of equilibrium may also arise. We have already alluded to the possibility of a roof-edge equilibrium, denoted by $\left(x_{1 r}, x_{2 r}\right)$ and satisfying $0<x_{1 r}<1,0<x_{2 r}<1, x_{1 r}+x_{2 r}=1$; it exists whenever $(\alpha, \theta)$ lies in region i of Figure 1(a), but it is always an unstable saddle point (Appendix D). From Appendix $\mathrm{D}$, either one or two "base-edge" equilibria of the form $\left(x_{1 b}^{ \pm}, 0\right)$ with $0<x_{1 b}^{ \pm}<1$ 
(a) $\alpha=0.6, \theta=0.6, \rho=0, \lambda \rightarrow \infty, k=0$

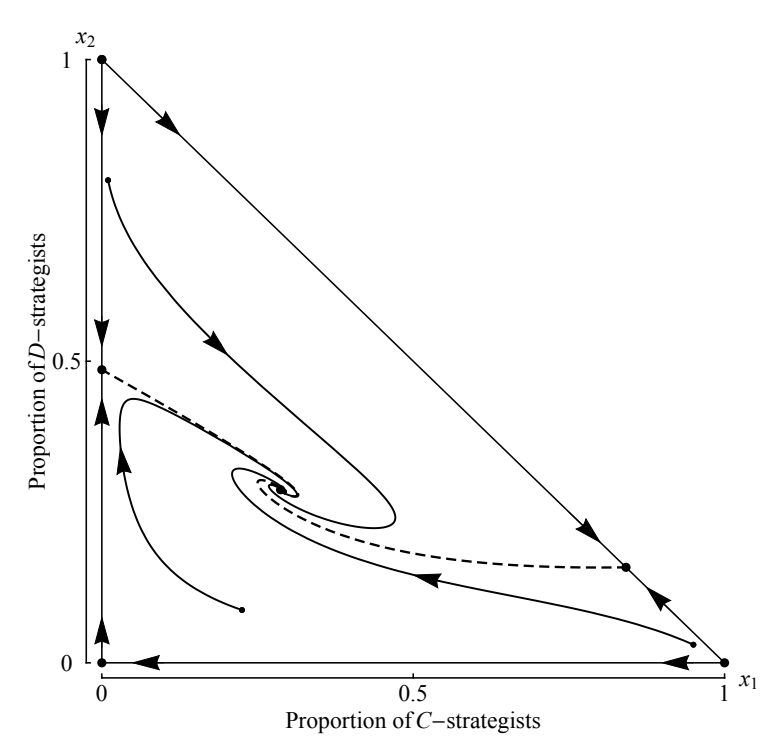

(b) $\alpha=0.43, \theta=0.49, \rho=0, \lambda \rightarrow \infty, k=0$

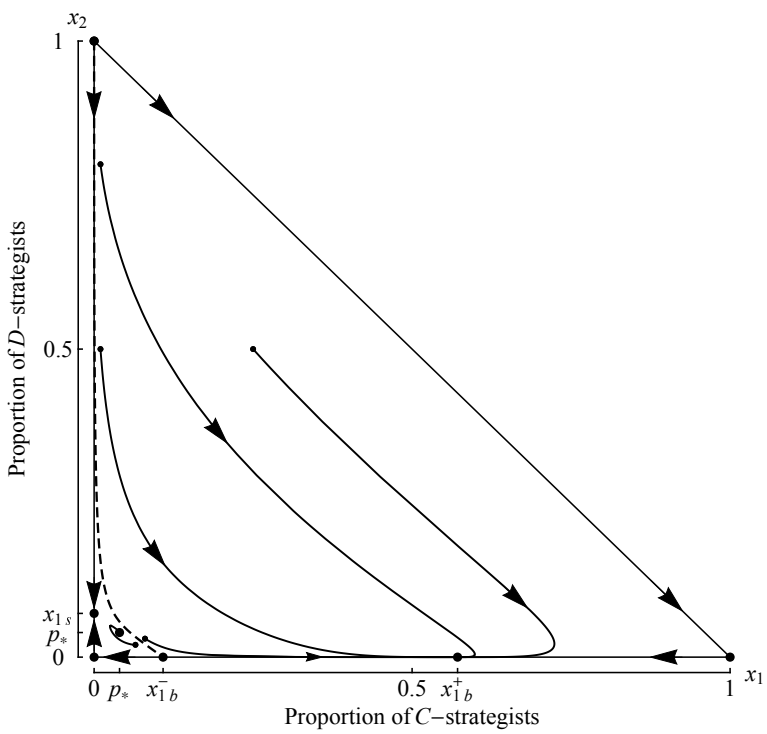

Figure 3: Phase-plane triangles $\Delta$ corresponding to two different points in region IIa of Figure 2(b). Large dots denote equilibrium points, small dots denote points at which sample trajectories begin. (a) This phaseplane triangle corresponds to the dot in region IIa $\cap \overline{\text { iii }}$ of 2(b), where an overbar denotes a set complement. There are six equilibrium points: saddle points at $(0,0),(0,0.4858)$ and $(0.8417,0.1583)$, unstable nodes at $(1,0)$ and $(0,1)$ and a stable focus at $(0.2869,0.2869)$. Three sample trajectories are shown, with initial points $(0.01,0.8),(0.95,0.03)$ and $(0.225,0.0875)$. Separatrices join the saddle points at $(0,0.4858)$ and $(0.8417,0.1583)$ to the stable focus at $(0.2869,0.2869)$. Because this focus is the only local attractor, it is also the global attractor: the population evolves to a polymorphism of all three strategies. (b) This phase-plane triangle corresponds to the dot in region IIa $\cap$ iii of $2(b)$. There are seven equilibrium points: saddle points at $(0,0),(0,0.07099)(0.1084,0)$ and $(1,0)$, an unstable node at $(0,1)$, a stable focus at $(0.03994,0.03994)$ and a stable node at $(0.5715,0)$. Five sample trajectories are shown, with initial points $(0.01,0.8),(0.01,0.5)$, $(0.25,0.5),(0.065,0.02)$ and $(0.08,0.03)$. To avoid clutter, no arrows were placed on the last two trajectories, which converge to the stable focus and the stable node, respectively. A separatrix, shown dashed, joins the unstable node at $(0,1)$ to the saddle point at $(0.1084,0)$. Because there are now two local attractors, there is no longer a global attractor; rather, $\left(x_{1}(t), x_{2}(t)\right.$ converges to $(0.03994,0.03994)$ from points on the left of the separatrix and to $(0.5715,0)$ from points on the right of it. That is, the population evolves to a polymorphism of all three strategies or a monomorphism of $C$, according to whether $\left(x_{1}(0), x_{2}(0)\right.$ lies on the left or the right of the separatrix. 
will arise when $(\alpha, \theta)$ lies in region iii $\cup$ iv $\cup$ vi of Figure 1(a). Region iv corresponds to a base-edge saddle point; region vi corresponds to a base-edge stable node; and region iii corresponds to two base-edge equilibria, a saddle point and a stable node. Again from Appendix D, a "side-edge" equilibrium of the form $\left(0, x_{2 s}\right)$ with $0<x_{2 s}<1$ arises when $(\alpha, \theta)$ lies in region $\mathrm{i} \cup \mathrm{ii} \cup \mathrm{iii} \cup \mathrm{iv}$ of Figure 1(a) or, equivalently, region I $\cup$ II of Figure 1(b); but it is always an unstable saddle point. Finally, for all $(\alpha, \theta)$ in the very same region of $S$, an interior equilibrium invariably arises on the $45^{\circ}$ open line segment

$$
\Lambda=\left\{\left(x_{1}, x_{2}\right) \mid 0<x_{1}=x_{2}<\frac{1}{2}\right\}
$$

joining $(0,0)$ to $\left(\frac{1}{2}, \frac{1}{2}\right)$ in $\Delta$. It is always either a node or a focus; but it is an attractor only for $(\alpha, \theta) \in \mathrm{Ia} \cup \mathrm{IIa}$. For $(\alpha, \theta) \in \mathrm{Ib} \cup \mathrm{IIb} \cap$ iii $\cup$ iv there is a local attractor on the boundary

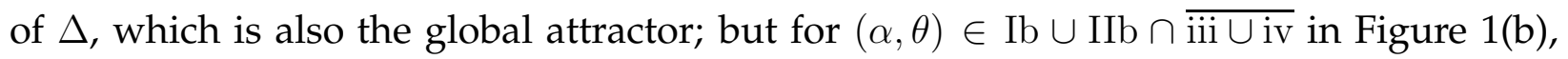
no static local attractor exists. In this region, $\left(x_{1}(t), x_{2}(t)\right.$ converges to a limit cycle, as illustrated by Figure 4 .

The result is that a unique global attractor exists for all $(\alpha, \theta) \in S$ except points lying in the narrow transitional region $C$ in Figure 5. In this region, strategy $D$ persists only if the initial proportion of strategy $C$ is very low (so that $\left(x_{1}(0), x_{2}(0)\right)$ lies to the left of the separatrix discussed in Appendix D). For all $(\alpha, \theta)$ in region $D \cup E$ of Figure 5, however, strategy $D$ is guaranteed to persist at some level, either as part of a static polymorphism (region $D$ ) or as part of a periodic dynamic equilibrium or limit cycle that surrounds an unstable equilibrium (region $E$ ). Because $p_{*}$ is the average value of $x_{1}(t)$ or $x_{2}(t)$ over the period of any such limit cycle, $p_{*}$ is a suitable measure of the representation of strategy $D$ (or $C$ ) within a polymorphism of all three strategies, regardless of whether strategy $D$ persists statically or a dynamically. We therefore refer to $p_{*}$ as the "strength" of strategy $D$ in such a polymorphism.

Within region $\mathrm{E}$, the further $(\alpha, \theta)$ moves away from the boundary with region $D$ and into the region where the equilibrium at $\left(p_{*}, p_{*}\right)$ is unstable, the greater the amplitude of the limit cycle; thus its amplitude increases with $\theta$ and decreases with $\alpha$. The nearer $(\alpha, \theta)$ moves to the boundary with region $\mathrm{B}$, the more the limit cycle approaches the side edge of $\Delta$ where $x_{1}=0$. So it is possible that a stochastic perturbation to strategy $C$ could drive it to extinction, but only at a point where the proportion of strategy $D$ in the dynamic polymorphism is high. Then $\left(x_{1}(t), x_{2}(t)\right.$ would move along $x_{1}=0$ towards the saddle point at $\left(0, x_{2 s}\right)$; this is the only circumstance in which this equilibrium would become an attractor. However, a single $C$ mutant would shift $\left(x_{1}(t), x_{2}(t)\right.$ back onto the limit cycle. So strategy $D$ persists at strength $p_{*}$ for any $(\alpha, \theta)$ in region $\mathrm{D} \cup \mathrm{E}$, regardless of whether its proportion in the strategy mix is fixed or changes with time. For any given value of $\theta$, 
(a) $\alpha=0.5, \theta=0.8, \rho=0, \lambda \rightarrow \infty, k=0$

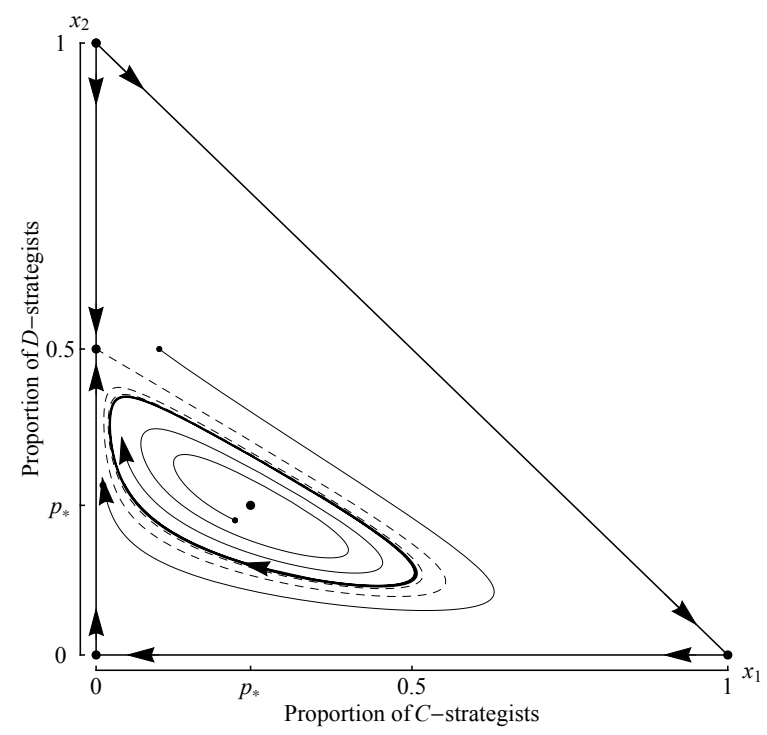

(b) $\alpha=0.5, \theta=0.8, \rho=0, \lambda \rightarrow \infty, k=5 . \times 10^{-7}$

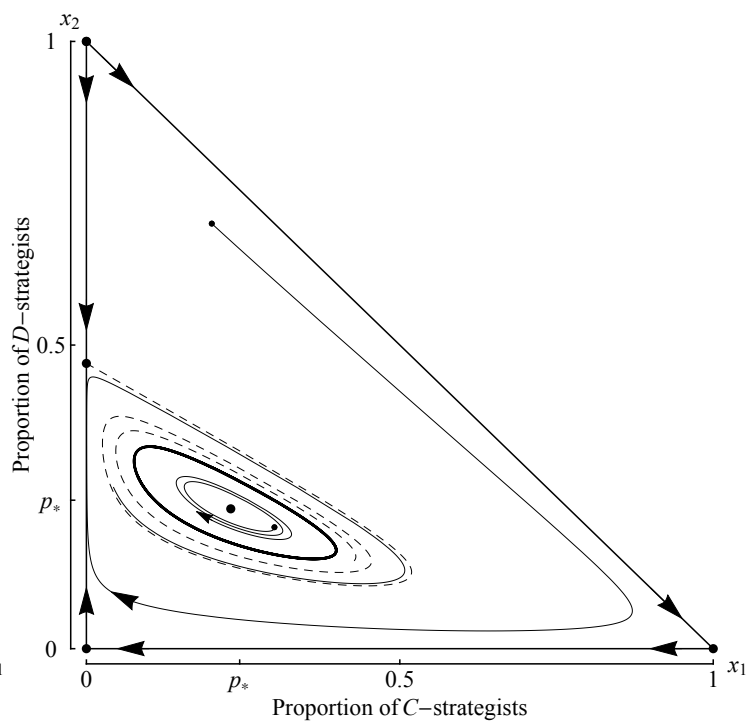

Figure 4: Examples of persistence of strategy $D$ in a limit cycle. The phase-plane triangle $\Delta$ is shown for $\alpha=0.5, \theta=0.8, \rho=0, \lambda \rightarrow \infty$ and two values of $k$; as in Figures 2 and 3, large dots denote equilibrium points, small dots denote points at which sample trajectories begin. In both cases, there are five equilibrium points: saddle points at $(0,0)$ and $\left(0, x_{2 s}\right)$, an unstable node at $(0,1)$, an unstable focus at $\left(p_{*}, p_{*}\right)$ and a nonhyperbolic saddle-node equilibrium at $(1,0)$, as discussed in Appendix D. The closed curve is a limit cycle surrounding the unstable focus at $\left(p_{*}, p_{*}\right)$. Two sample trajectories of approach are shown, one starting inside the limit cycle and one starting outside it; for simplicity of illustration, they are not sketched all the way to convergence. Also shown dashed is the separatrix from the saddle point at $\left(0, x_{2 s}\right)$ towards the limit cycle. (a) Here $k \rightarrow 0$. This phase-plane triangle $\Delta$ corresponds to the point $(\alpha, \theta)=(0.5,0.8)$ in region IIb $\cap \overline{\text { iii }} \cap \overline{\text { iv }}$ of Figure 2(b); $x_{2 s}=0.5$ and $p_{*} \approx 0.2445$. (b) Here $k=5 \times 10^{-7} ; x_{2 s} \approx 0.4698$ and $p_{*} \approx 0.2303$. In both cases, the limit cycle is a global attractor from inside $\Delta$ : the population evolves to a dynamic equilibrium, a periodic polymorphism of all three strategies. 


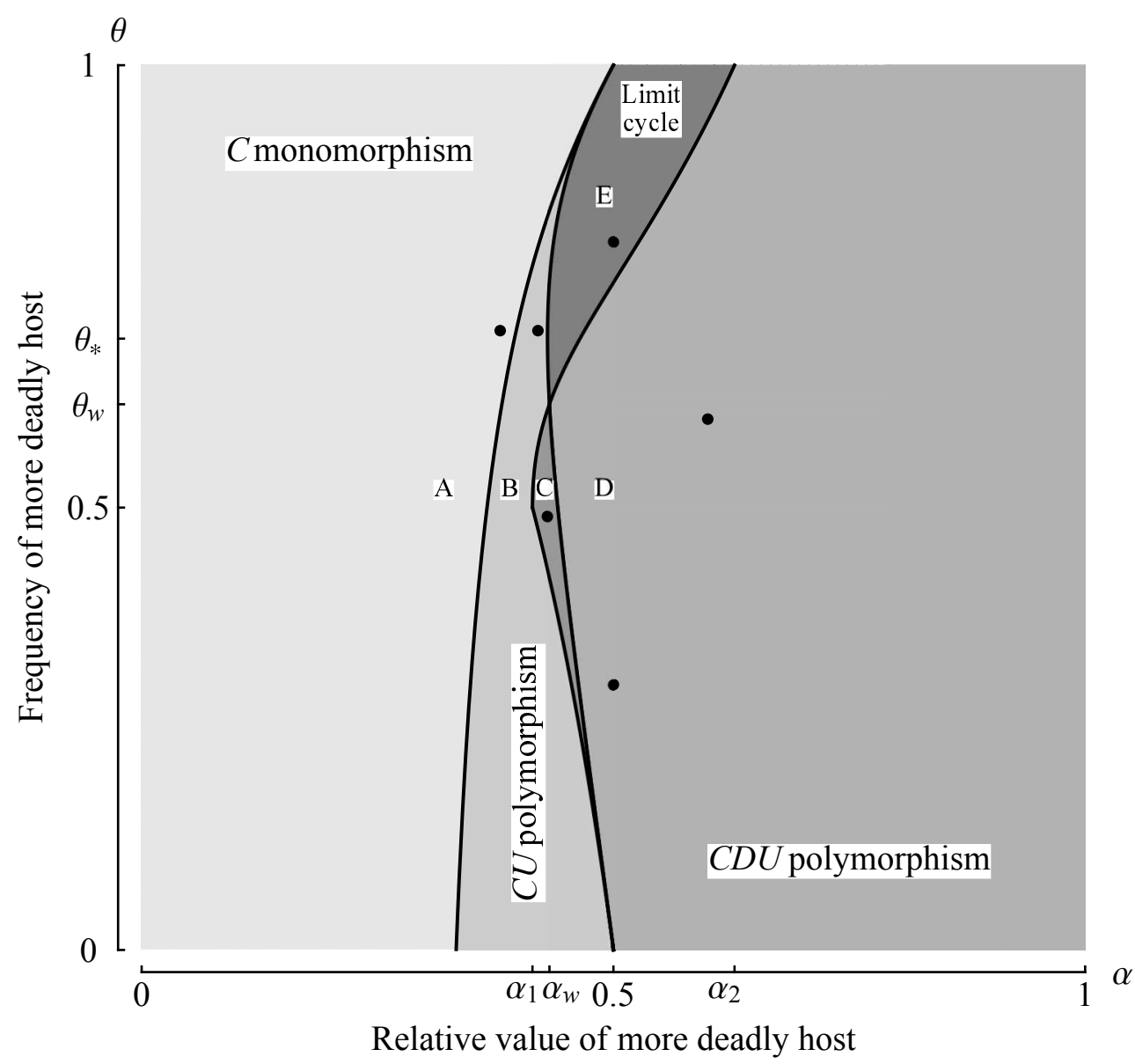

Figure 5: Strategy mix to which the population evolves as a function of $\alpha$ and $\theta$ for $\rho=0, \lambda \rightarrow \infty$ and $k=0$. For points $(\alpha, \theta)$ in region $\mathrm{A},\left(x_{1}(t), x_{2}(t)\right)$ is attracted to the vertex $(1,0)$ in the bottom right-hand corner of the phase triangle $\Delta$, so the population evolves to a monomorphism of strategy $C$. For points in region $\mathrm{B},\left(x_{1}(t), x_{2}(t)\right)$ is attracted to the base-edge of $\Delta:\left(x_{1}(\infty), x_{2}(\infty)\right)=\left(x_{1 b}^{+}, 0\right)$ where $x_{1 b}^{+}$is defined by (D.10), a polymorphism of strategies $C$ and $U$. Only for points $(\alpha, \theta)$ in regions $\mathrm{D}$ or $\mathrm{E}$ is strategy $D$ guaranteed to persist. For $(\alpha, \theta)$ in region $\mathrm{D},\left(x_{1}(t), x_{2}(t)\right)$ is attracted to a stable node or focus; whereas for $(\alpha, \theta)$ in region $\mathrm{E}$, the population evolves to a limit cycle instead. In either case, the population evolves to a (static or dynamic) polymorphism of all three strategies. In the narrow transitional region $C$, the population evolves either to a static polymorphism of all three strategies or to $\left(x_{1 b}^{+}, 0\right)$, according to whether $\left(x_{1}(0), x_{2}(0)\right)$ lies to the left or the right of a separatrix joining the unstable node at $(0,1)$ to the saddle point at $\left(x_{1 b}^{-}, 0\right)$. The dots in regions A, B, C and E indicate the points for which the phase trajectories are plotted in Figures 2(a), 2(b), 3(b), and 4(a), respectively; in region D, the upper dot corresponds to Figure 3(a) and the lower dot to Figure $6(\mathrm{a})$. The points $\left(\alpha_{*}, \theta_{*}\right) \approx(0.4302,0.691)$ and $\left(\alpha_{w}, \theta_{w}\right) \approx(0.4322,0.6168)$ are, respectively, the point at which the boundary between regions $\mathrm{B}$ and $\mathrm{E}$ has a vertical tangent and the lowest point of the wedge-shaped region $E ; \alpha_{*}$ is not marked on the horizontal axis because it is too close to $\alpha_{w}$. See Appendix D for further details. 
within region $\mathrm{D} \cup \mathrm{E}$, the least value of $\alpha$ is $\{\sqrt{1-\theta}-1+\theta\} / \theta$; as $\alpha$ increases from that value to 1 , the strength of strategy $D$ increases from 0 to $\frac{1}{2}$.

How likely is such a limit cycle to arise in practice? There are currently no empirical data to address this question. Nevertheless, from inspection of Figure 5, we can posit that a necessary condition for the existence of such a limit cycle is that $\alpha_{*}<\alpha<\alpha_{2}$ and $\theta_{w}<\theta<1$, or $0.432<\alpha<0.628$ and $0.617<\theta<1$ approximately.

We conclude this section by noting that the proportion of large individuals in a polymorphic population is always higher than the strength of strategy $D$ because a $U$-strategist is large with probability $\omega_{L}$. Let $P_{L}$ and $P_{N}$ denote the proportions of individuals in the population who are large and normal, respectively. Then

$$
P_{L}=x_{2}+\omega_{L} x_{3}, \quad P_{N}=x_{1}+\omega_{N} x_{3}
$$

where $\omega_{L}$ and $\omega_{N}$ are defined by (1), $x_{3}$ is determined by (11) and $P_{L}+P_{N}=1$. Thus, for example, at the static polymorphism in Figure 3(a) where $D$ persists at strength $p_{*} \approx 0.287$, the proportion of individuals in the population who are large is $p_{*}+\omega_{L}\left(1-2 p_{*}\right) \approx 0.489$. An important point for future empirical investigations is that an ESS may not itself be directly observable (Mesterton-Gibbons, 2019, p. 360); and in this context, $P_{L}$ and $P_{N}$ are observable, whereas $p_{*}$ is not.

\section{Implications of owner advantage and other departures from the reduced model}

In this section we relax some of the assumptions of the previous section by discussing the effects of owner advantage $(\rho \neq 0)$ and of reduced parasitoid density $(a<\infty$ or $k>0)$ or size advantage $(\lambda<\infty)$. Our results were obtained largely from numerical exploration of the larger five-dimensional parameter space, although some analysis was possible. Details appear in Appendix E. Here we confine ourselves to a brief summary, especially for $k$ and $\lambda$, so that we can focus on the more important implications of increasing $\rho$.

The effects of reducing parasitoid density and of reducing size advantage are similar. In essence, for sufficiently large $\alpha$, strategy $D$ will persist at reduced parasitoid density as long as $k$ does not depart too greatly from its very small value in the reduced model, when $\rho$ and $\lambda$ are unchanged; and again for sufficiently large $\alpha$, strategy $D$ will persist at reduced size advantage as long as $\lambda$ does not depart too greatly from its very large value in the reduced model, when $\rho$ and $k$ are unchanged. Both of these results are strongly intuitive: size advantage in contests is less likely to be favoured if a contest is less likely in the first place, or if it is less than certain to be decisive when a contest does arise. Either increasing $k$ or decreasing $\lambda$ from its reduced-model value reduces the strength of strategy 
$D$ in the polymorphism that allows it to persist, and causes the interior equilibrium $\left(p_{*}, p_{*}\right)$ to drift down $\Lambda$ towards the origin, where it eventually disappears (along with strategy $D)$. Significantly, however, in the absence of owner advantage $(\rho=0)$, whenever an interior equilibrium exists, it always lies in $\Lambda$.

By contrast, as soon as owner advantage enters the picture $(\rho \neq 0)$, the interior equilibrium veers off $\Lambda$ towards the side edge of $\Delta$, so we must now instead denote it by $\left(p_{*}, q_{*}\right)$, where $p_{*}<q_{*}$. The effect is illustrated by Figure 6 . The first panel shows a reducedmodel phase triangle for $(\alpha, \theta)$ in region $\mathrm{D}$ with a lower value of $\theta$ than in Figure 3(a), so that the global attractor is a stable node as opposed to a stable focus. Both strategy $C$ and strategy $D$ have strength $p_{*} \approx 0.113$ in the corresponding polymorphism of all three strategies. Steadily increasing the value of $\rho$ from zero increases the strength of $D$ in the polymorphism, but decreases that of both $C$ and $U$. The second panel shows the phase plane at $\rho=0.2$. The stable node has veered off $\Lambda$ to $\left(p_{*}, q_{*}\right) \approx(0.0766,0.211)$; and so the strength of $D$ has increased by almost 0.1 , while that of $C$ and that of $U$ have decreased by almost 0.04 and over 0.06 , respectively. As $\rho$ continues to increase, $\left(p_{*}, q_{*}\right)$ moves steadily towards the side-edge saddle point, which it absorbs at $\left(p_{*}, q_{*}\right) \approx(0,0.279)$ for $\rho \approx 0.523$. For this and any higher value of $\rho$, the global attractor is a side-edge stable node, as illustrated by the third panel of Figure 6 (and the fourth panel is essentially unrelated to the present discussion, being included to illustrate a point about the effect of $k$ in Appendix E). Strategy $C$ has now been eliminated from the polymorphism, but $D$ persists alongside $U$, whose strength is lower than for $\rho=0$ (although still much higher than that of $D$ ).

Figure 6 illustrates that owner advantage reinforces the effect of size advantage to increase the strength of strategy $D$ in a polymorphism where $D$ would have persisted for $\rho=0$, because $(\alpha, \theta)$ lies in region D of Figure 5. However, a more interesting question is whether owner advantage can enable $D$ to persist in region A of Figure 5 , where $D$ would not persist for $\rho=0$. The answer is yes. What happens in this case is that increasing $\rho$ induces the globally attracting stable node at $(1,0)$ to migrate leftward along $x_{2}=0$ toward the origin, and then upward along the side edge. This effect is illustrated by Figure 7 for $\alpha=0.25$ and $\theta=0.5$, so that $(\alpha, \theta)$ lies in region $\mathrm{A}$ of Figure 5. In this case, $\left(x_{1}(t), x_{2}(t)\right)$ transitions away from $(1,0)$ and through the origin at $\rho \approx 0.25$ and $\rho \approx 0.467$, respectively.

To appreciate how significantly owner advantage changes the overall picture, we need only remember that in its absence a global attractor on the side edge of $\Delta$ is impossible. For $\rho=0$, no $(\alpha, \theta)$ in region $\mathrm{v} \cup$ vi of Figure 1 ever corresponds to a side-edge equilibrium; and when a side-edge equilibrium does exist, it is always an unstable saddle point. By contrast, Figures 7 and 8 show that a point in region v of Figure 1 can correspond not only 


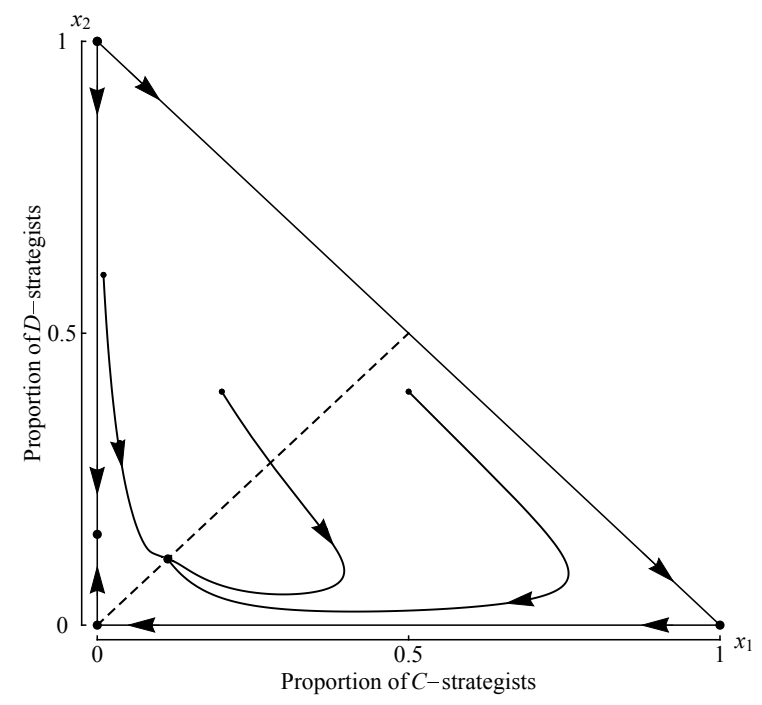

(c) $\alpha=0.5, \theta=0.3, \rho=0.6, \lambda \rightarrow \infty, k=0$

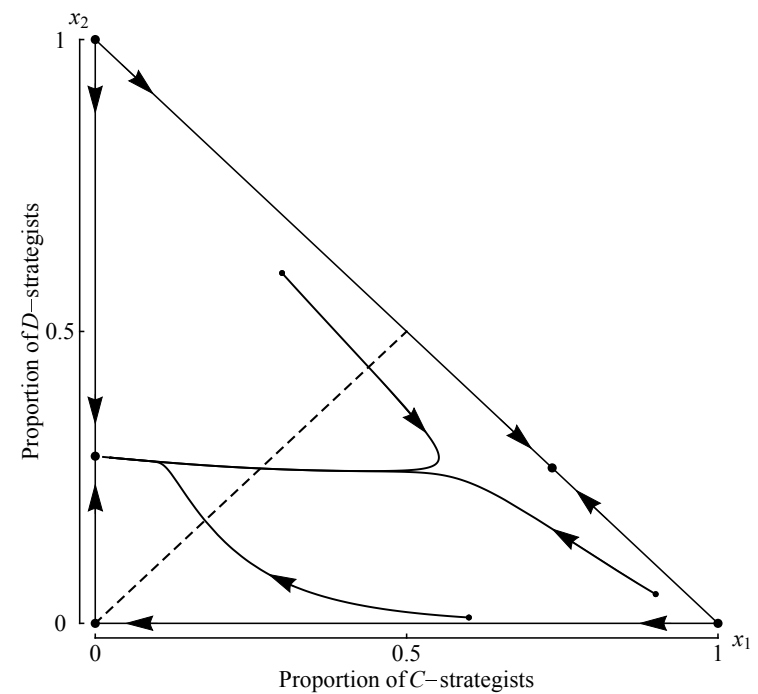

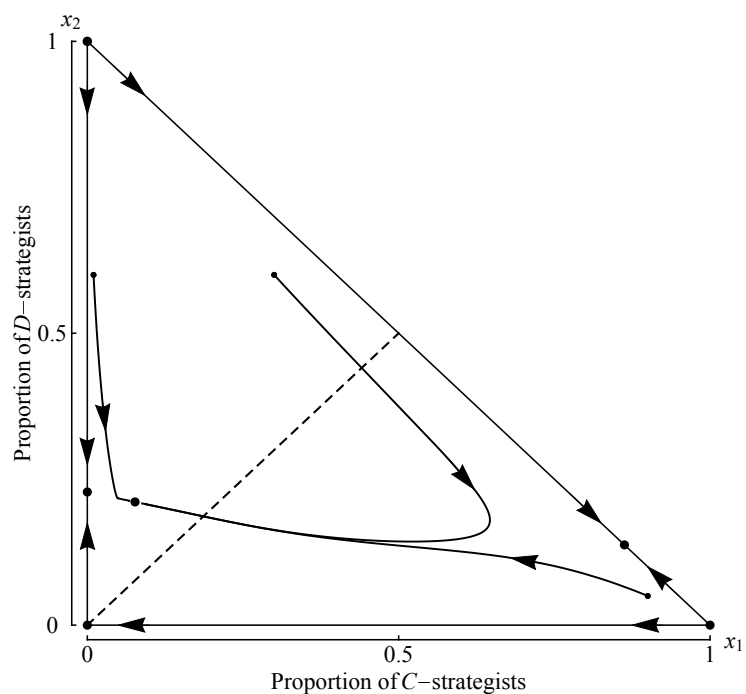

(d) $\alpha=0.5, \theta=0.3, \rho=0.2, \lambda \rightarrow \infty, k=0.01$

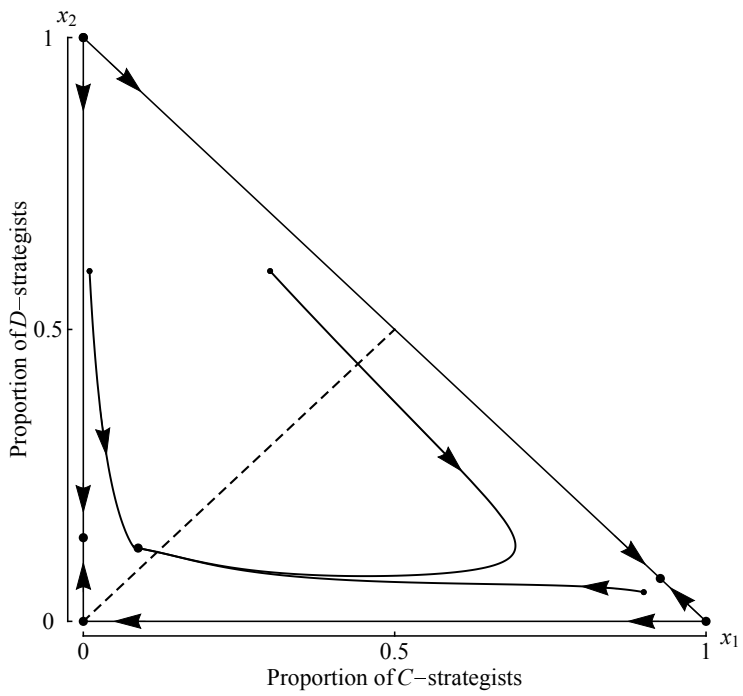

Figure 6: Phase-plane triangles $\Delta$ corresponding to the lower point marked in region D of Figure 5 for different values of $\rho$. The line segment $\Lambda$ defined by (17) is shown dashed; as in Figures 2-4, large dots denote equilibrium points, small dots denote points at which sample trajectories begin. In addition to the three vertex equilibria, there is a side-edge equilibrium in each example, a roof-edge saddle point in in each example except (a), and an interior equilibrium in in each example except (c). In (a), (b) and (d), the interior equilibrium is a globally attracting stable node; in (c), the global attractor has migrated to the side-edge; and in (a), (b) and (d), the side-edge equilibrium is a saddle point. See $\S 4$ and Appendix E for further details. 
to a side-edge equilibrium, but also to a global attractor if $\rho$ is sufficiently large. In Figure 8 , we have $\operatorname{set} \theta=\frac{1}{2}$ (equal numbers of natural and more deadly hosts) to match Figure 7 and we have replaced $S$ by the new parameter square

$$
\tilde{S}=\{(\alpha, \rho) \mid 0 \leq \alpha, \rho \leq 1\}
$$

to better illustrate the effect of varying $\alpha$ and $\rho$ while holding $\theta$ fixed; if we think of $S$ in Figure 5 as the horizontal base of a cube with $\rho$ increasing vertically, then $\tilde{S}$ corresponds to a vertical cross section through the centre of this cube in the plane where $\theta=\frac{1}{2}$.

A very small value of $\alpha$ may require a very large value of $\rho$ to ensure the persistence of strategy $D$, and Figure 8 demonstrates its persistence for only a single value of $\theta$. Nevertheless, it is shown in Appendix $\mathrm{E}$ that $\rho$ can always be made sufficiently large to ensure that $D$ persists, although if $\alpha$ is small, $D$ may persist only if $\rho$ is close to 1 , and only at very low strength. However, the strength of $D$ is less important than the proportion of large individuals, who may be either $D$-strategists or $U$-strategists; and although, for example, the strength of $D$ at the global attractor in Figure 7(c) is only $q_{*} \approx 0.0729$, from (18) the proportion of large individuals in this polymorphism is $q_{*}+\omega_{L}\left(1-q_{*}\right) \approx 0.258$.

\section{Discussion}

Advancement of our understanding of animal contests has been possible through a successful combination of well-integrated theoretical and experimental approaches (Briffa \& Hardy, 2013; Kokko, 2013; Sherratt \& Mesterton-Gibbons, 2013). Game-theory is not only useful to predict how general strategies evolve in order to maximize fitness, but it is has been proven to be flexible enough to adapt towards the specific biological features of a given species of interest (Hammerstein \& Riechert, 1988). To date, limited attention has been given to the role played by anthropogenic disturbances, yet these are increasingly frequent components of the environment of free-living organisms as a result of globalization, leading to accidental introductions, and climate change affecting species' geographical ranges (Mooney \& Hobbs, 2000).

We have used evolutionary game theory (Broom \& Rychtář, 2013; Mesterton-Gibbons, 2019; Sigmund, 2010) to address the following question: can size advantage in contests among parasitoid wasps sustain a preference for a more deadly host? The question is currently a theoretical one because relevant empirical observations are so preliminary, and hence we have used a low-dimensional parameter space for clarity of prediction: our answer depends on only two dimensionless parameters, namely, the ratio $(\alpha)$ of the reproductive value of a more deadly host to that of a natural host and the proportion $(\theta)$ 
(a) $\alpha=0.25, \theta=0.5, \rho=0.2, \lambda \rightarrow \infty, k=0$

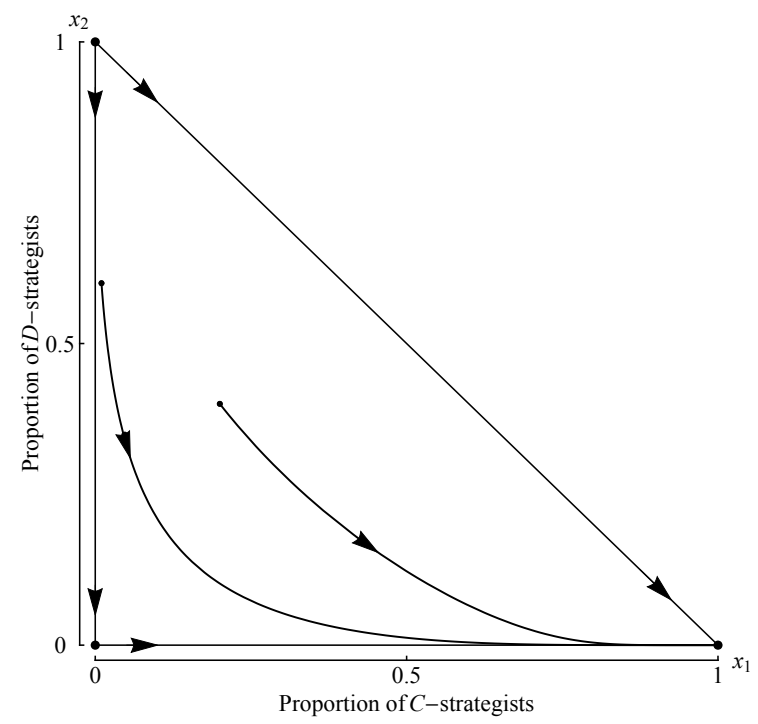

(c) $\alpha=0.25, \theta=0.5, \rho=0.6, \lambda \rightarrow \infty, k=0$

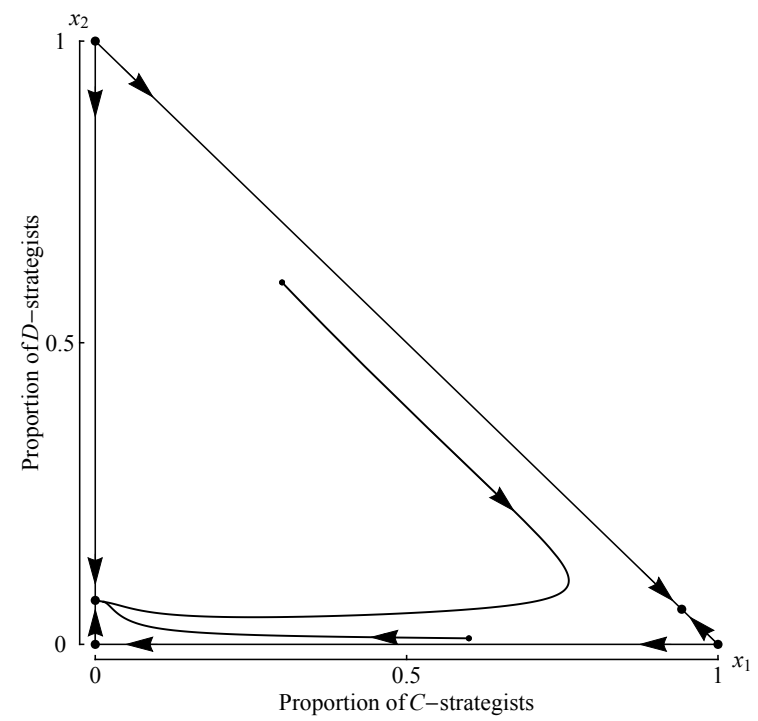

(b) $\alpha=0.25, \theta=0.5, \rho=0.4, \lambda \rightarrow \infty, k=0$

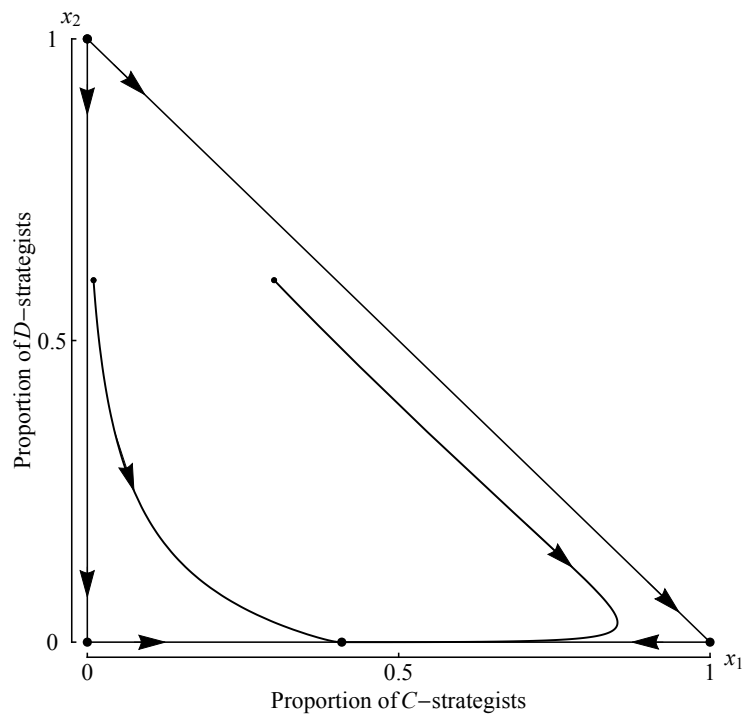

(d) $\alpha=0.25, \theta=0.5, \rho=0.8, \lambda \rightarrow \infty, k=0$

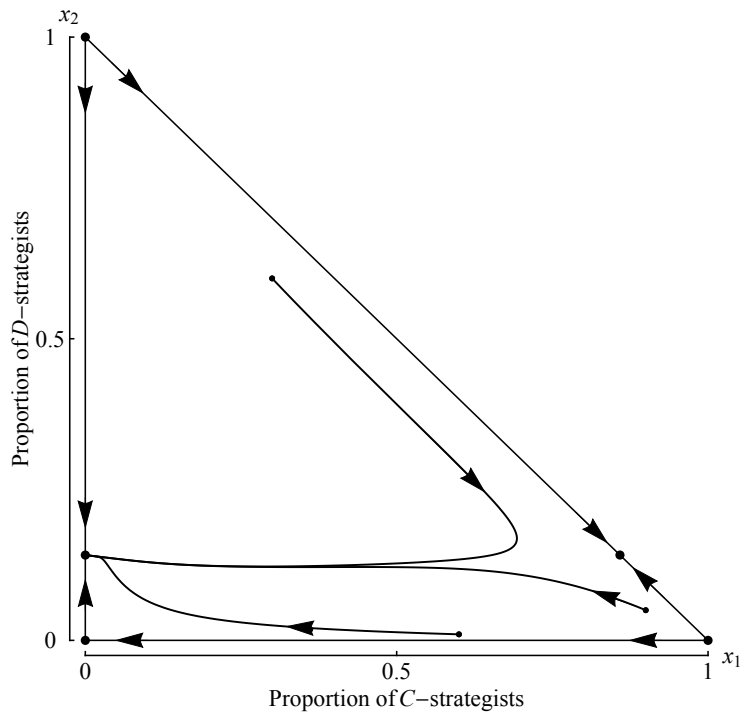

Figure 7: Phase-plane triangles $\Delta$ corresponding to a point in region A of Figure 5 for different values of $\rho$. As in Figures 2-4 and 6, large dots denote equilibrium points, small dots denote points at which sample trajectories begin. In addition to the three vertex equilibria, there is a base-edge stable node in (b), and there are both a roof-edge saddle point and a side-edge stable node in both (c) and (d). As $\rho$ increases, the globally attracting stable node migrates leftward along $x_{2}=0$ toward the origin, and then upward along the side edge, transitioning away from $(1,0)$ and through the origin at $\rho \approx 0.25$ and $\rho \approx 0.467$, respectively. 


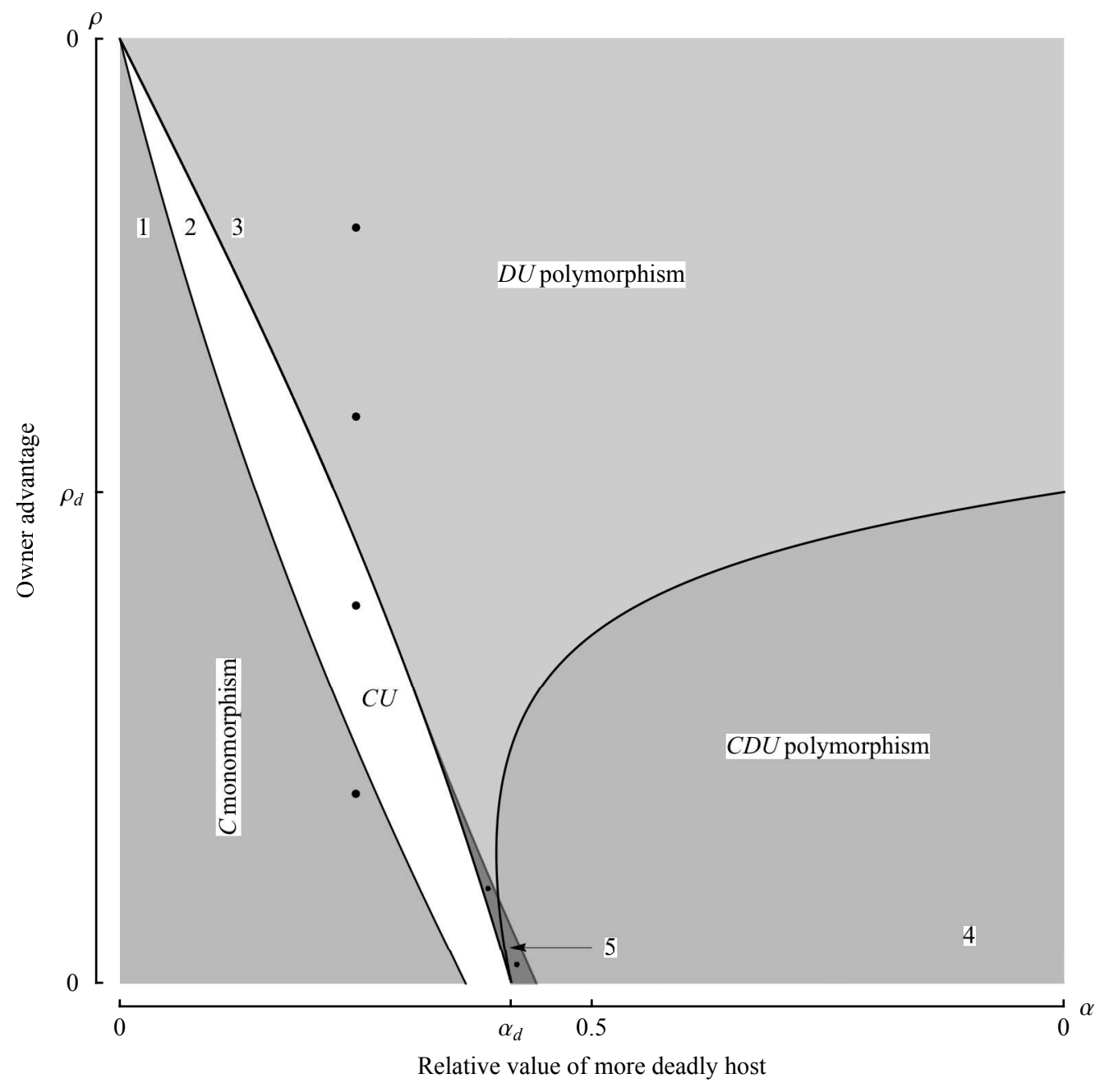

Figure 8: Strategy mix to which the population evolves as a function of $\alpha$ and $\rho$ for $\theta=\frac{1}{2}, \lambda \rightarrow \infty$ and $k=0$. For points $(\alpha, \rho)$ in region 1 of $\tilde{S}$ (defined by (19)), $\left(x_{1}(t), x_{2}(t)\right)$ is attracted to vertex $(1,0) \in \Delta$ as $t \rightarrow \infty$, so the population evolves to a monomorphism of strategy $C$. For points in unshaded region 2, $\left(x_{1}(\infty), x_{2}(\infty)\right)=\left(x_{1 b}^{+}, 0\right)$ on the base edge of $\Delta$, so the population evolves to a polymorphism of strategies $C$ and $U$. Only for points $(\alpha, \rho)$ in region 3 or region 4 and outside transitional region 5 is strategy $D$ guaranteed to persist, within a polymorphism of either $D$ and $U$ or all three strategies, as indicated. For points in region $3,\left(x_{1}(\infty), x_{2}(\infty)\right)=\left(0, x_{2 s}\right)$ on the side edge of $\Delta$, except in the intersection of regions 3 and 5 , where $\left(x_{1}(\infty), x_{2}(\infty)\right)=\left(0, x_{2 s}\right)$ only if $\left(x_{1}(0), x_{2}(0)\right)$ is very close to $\left(0, x_{2 s}\right)$, and otherwise $\left(x_{1}(\infty), x_{2}(\infty)\right)=\left(x_{1 b}^{+}, 0\right)$ as in the rest of region 3 . For points in region $4,\left(x_{1}(\infty), x_{2}(\infty)\right)=\left(p_{*}, q_{*}\right)$ in the interior of $\Delta$, except in the intersection of regions 4 and 5 , where $\left(x_{1}(\infty), x_{2}(\infty)\right)=\left(p_{*}, q_{*}\right)$ only if $\left(x_{1}(0), x_{2}(0)\right)$ is very close to $\left(p_{*}, q_{*}\right)$, and otherwise $\left(x_{1}(\infty), x_{2}(\infty)\right)=\left(x_{1 b}^{+}, 0\right)$ as in the rest of region 5 . The larger dots at $(0.25,0.2),(0.25,0.4),(0.25,0.6)$ and $(0.25,0.8)$ correspond to panels $(a),(b),(c)$ and $(d)$, respectively, in Figure 7. Note that moving along the base of the above square from left to right corresponds to moving in the same direction along the central axis $\theta=\frac{1}{2}$ of the square in Figure 5. For all other details, including the purpose of the smaller dots in region 5, see Appendix G. 
of all hosts that are of the more deadly variety. And because the left-hand boundary of region $\mathrm{E}$ in Figure 5 has its vertical tangent at $\left(\alpha_{*}, \theta_{*}\right)$, where $\alpha_{*} \approx 0.4302$ and $\theta_{*} \approx 0.691$, our analysis predicts that the answer is yes, but that $D$ can persist only if the value of a more deadly host is at least $43 \%$ of the value of a natural one (though, on the other hand, strategy $D$ is also guaranteed to exist, even at low proportions of the more deadly host, if the relative value is $50 \%$ or greater, because $(\alpha, \theta) \in \mathrm{D} \cup \mathrm{E}$ in Figure 5 for all $\left.\alpha \geq \frac{1}{2}\right)$.

The two-parameter model we have analysed in $\S 3$ is a special case of the more general five-parameter model that we formulated in $\S 2$ (and Appendix A), obtained in the limit of no owner advantage, extreme size advantage and high parasitoid density, that is, for $\rho=0, \lambda \rightarrow \infty$ and $k=0$. In principle, we could repeat our analysis for any other set of values of the other three parameters, that is, for $\rho>0$, finite $\lambda$ and $k>0$, and for each such choice we could in principle obtain analogues of Figures 1-5. In practice, however, such an investment of current effort would be unwise, because in the absence of empirical data, no such values have any greater significance than the limiting values we have already chosen.

Nevertheless, we have explored the more general five-dimensional parameter space numerically, as described in $\S 4$, and in particular have investigated how owner advantage can reinforce the advantage of size. Our analysis not only shows that a preference for the more deadly host is most favoured when the asymmetries of size and ownership both apply, but it also predicts that an obligate preference for the natural host cannot be eliminated unless both asymmetries operate. This result is implicit in Figures 6 and 7 but is further clarified by the analytical results presented in Appendix F. Because owner advantage significantly reduces the value of $\alpha$ at which a preference for the more deadly host can persist, it seems that size advantage in contests is unlikely by itself to sustain a preference for Halyomorpha hyalis among Trissolcus basalis, but that such a preference could be maintained by the simultaneous operation of both size advantage and ownership advantage (as is observed in some parasitoids, Hardy et al., 2013). However, the issue remains very much an open question because empirical studies are at such a preliminary stage, even though some aspects of patch defence and contest behaviour in T. basalis have been previously investigated with regard to its role as a parasitoid of the pentatomid bug Agonoscelis rutila (Fabricious) (Field \& Calbert, 1998; Field, 1998; Field et al., 1998; Field \& Calbert, 1999). Although asymmetries in wasps' contestant abilities were not explored, this work indicates that the main factor influencing contest outcome is prior ownership, closely fitting the Bourgeois strategy that was explored by classic game-theoretic models during the early development of general theory for contests: i.e., females that arrive first on the patch almost always win against subsequent intruders (Field et al., 1998). 
The oviposition into invasive hosts by indigenous parasitoids would be expected to select for an enhanced ability to survive development in those hosts and thus an evolutionary change in $\alpha$, the relative reproductive value of the unnatural host (Berthon, 2015). Hence, it is possible that the contest biology of T. basalis will act to promote a more stable association with the Brown Marmorated Stink Bug, ultimately facilitating escape from what has been seen as the evolutionary trap of accepting hosts that are unsuitable for the development of its offspring (Schlaepfer et al., 2002, 2005; Abram et al., 2014). The invasive Brown Marmorated Stink Bug is a major agricultural pest in Europe and in north America where the biological control provided by resident egg parasitoids such as T. basalis is generally considered insufficient to reduce the pest population density below economical thresholds (Rice et al., 2014; Leskey \& Nielsen, 2018). An enhanced ability of resident natural enemies to survive development in this pest may indicate that, over generations, the value of native egg parasitoids in biological control could increase.

\section{References}

Abram, P. K., Gariepy, T. D., Boivin, G., \& Brodeur, J. (2014). An invasive stink bug as an evolutionary trap for an indigenous egg parasitoid. Biological Invasions, 16, 1387-1395.

Abram, P. K., Hoelmer, K. A., Acebes-Doria, A., Andrews, H., Beers, E. H., Bergh, J. C., Bessin, R., Biddinger, D., Botch, P., Buffington, M. L., Cornelius, M. L., Costi, E., Delfosse, E. S., Dieckhoff, C., Dobson, R., Donais, Z., Grieshop, M., Hamilton, G., Haye, T., Hedstrom, C., Herlihy, M. V., Hoddle, M. S., Hooks, C. R. R., Jentsch, P., Joshi, N. K., Kuhar, T. P., Lara, J., Lee, J. C., Legrand, A., Leskey, T. C., Lowenstein, D., Maistrello, L., Mathews, C. R., Milnes, J. M., Morrison III, W. R., Nielsen, A. L., Ogburn, E. C., Pickett, C. H., Poley, K., Pote, J., Radl, J., Shrewsbury, P. M., Talamas, E., Tavella, L., Walgenbach, J. F., Waterworth, R., Weber, D. C., Welty, C., \& Wiman, N. G. (2017). Indigenous arthropod natural enemies of the invasive brown marmorated stink bug in North America and Europe. Journal of Pest Science, 90, 1009-1020. doi:10.1007/s10340-017-0891-7.

Aragón, F. J., Goberna, M. A., López, M. A., \& Rodríguez, M. M. L. (2019). Nonlinear Optimization. New York: Springer.

Balusu, R. R., Talamas, E. J., Cottrell, T. E., Toews, M. D., Blaauw, B. R., Sial, A. A., Buntin, D. G., Fadamiro, H. Y., \& Tillman, G. (2019). First record of Trissolcus basalis (hymenoptera: Scelionidae) parasitizing Halyomorpha halys (hemiptera: Pentatomidae) in the united states. Biodiversity Data Journal, 7, e39247. doi:10.3897/BDJ.7.e39247. 
Berthon, K. (2015). How do native species respond to invaders? mechanistic and trait based perspectives. Biological Invasions, 17, 2199-2211. doi:10.1007/s10530-015-0874-7.

Bertsekas, D. (2016). Nonlinear Programming. (3rd ed.). Belmont, Massachusetts: Athena Scientific.

Briffa, M., \& Hardy, I. C. W. (2013). Introduction to animal contests. In I. C. W. Hardy, \& M. Briffa (Eds.), Animal Contests (pp. 1-4). Cambridge: Cambridge University Press.

Broom, M., \& Rychtář, J. (2013). Game-Theoretical Models in Biology. Boca Raton, Florida: CRC Press.

Costi, E., Wong, W., Cossentine, J., Acheampong, S., Maistrello, L., Haye, T., Talamas, E. J., \& Abram, P. K. (2020). Variation in levels of acceptance, developmental success, and abortion of Halyomorpha halys eggs by native North American parasitoids. Biological Control, (p. in press). doi:10.1016/j.biocontrol.2020.104396.

Cusumano, A., Peri, E., Vinson, S. B., \& Colazza, S. (2011). Intraguild interaction between egg parasitoids exploiting host patches. Biocontrol, 56, 173-184.

Field, S. A. (1998). Patch Exploitation, Patch-leaving and Pre-emptive Patch Defence in the Parasitoid Wasp Trissolcus basalis (Insecta: Scelionidae). Ethology, 104, 323-338.

Field, S. A., \& Calbert, G. (1998). Patch defence in the parasitoid wasp Trissolcus basalis: when to begin fighting? Behaviour, 135, 629-642.

Field, S. A., \& Calbert, G. (1999). Don't count your eggs before they're parasitized: contest resolution and the trade-offs during patch defense in a parasitoid wasp. Behavioral Ecology, 10, 122-127. doi:10.1093/beheco/10.2.122.

Field, S. A., Calbert, G., \& Keller, M. A. (1998). Patch Defence in the Parasitoid Wasp Trissolcus basalis (Insecta: Scelionidae): The Time Structure of Pairwise Contests, and the 'Waiting Game'. Ethology, 104, 821-840.

Godfray, H. C. J. (1994). Parasitoids. Princeton, New Jersey: Princeton University Press.

Goubault, M., Mack, A. F. S., \& Hardy, I. C. W. (2007). Encountering competitors reduces clutch size and increases offspring size in a parasitoid with female-female fighting. Proceedings of the Royal Society of London B, 274, 2571-2577. 
Hammerstein, P., \& Riechert, S. E. (1988). Payoffs and strategies in territorial contests: ESS analyses of two ecotypes of the spider, Agelenopsis aperta. Evolutionary Ecology, 2, $115-138$.

Hardy, I. C. W., Goubault, M., \& Batchelor, T. P. (2013). Hymenopteran contests and agonistic behaviour. In I. C. W. Hardy, \& M. Briffa (Eds.), Animal Contests (pp. 147-177). Cambridge: Cambridge University Press.

Hardy, I. C. W., Griffiths, N. T., \& Godfray, H. C. J. (1992). Clutch size in a parasitoid wasp: a manipulation experiment. Journal of Animal Ecology, 61, 121-129.

Hofbauer, J., \& Sigmund, K. (1998). Evolutionary Games and Population Dynamics. Cambridge, UK: Cambridge University Press.

Karsai, I., Somogyi, K., \& Hardy, I. C. W. (2006). Body size, host choice and sex allocation in a spider hunting pompilid wasp. Biological Journal of the Linnean Society, 87, 285-296.

Kokko, H. (2013). Dyadic contests: modelling fights between two individuals. In I. C. W. Hardy, \& M. Briffa (Eds.), Animal Contests (pp. 5-32). Cambridge: Cambridge University Press.

Layek, G. C. (2015). An Introduction to Dynamical Systems and Chaos. New York: Springer.

Leskey, T. C., \& Nielsen, A. L. (2018). Impact of the invasive brown marmorated stink bug in North America and Europe: history, biology, ecology, and management. Annual Review of Entomology, 63, 599-618.

Mesterton-Gibbons, M. (2019). An Introduction to Game-Theoretic Modelling. (3rd ed.). Providence, Rhode Island: American Mathematical Society.

Mesterton-Gibbons, M., \& Hardy, I. C. W. (2004). The influence of contests on optimal clutch size: a game-theoretic model. Proceedings of the Royal Society of London B, 271, 971-978. doi:10.1098/rspb.2003.2670.

Mooney, H. A., \& Hobbs, R. J. (2000). Invasive Species in a Changing World. Washington, D. C.: Island Press.

Peri, E., Foti, M. C., Martorana, L., Cusumano, A., \& Colazza, S. (2020). The invasive stink bug Halyomorpha halys affects the reproductive success and the experience-mediated behavioural responses of the egg parasitoid Trissolcus basalis. Submitted to BioControl. 
Rice, K. B., Bergh, C. J., Bergmann, E. J., Biddinger, D. J., Dieckhoff, C., Dively, G., Fraser, H., Gariepy, T., Hamilton, G., Haye, T., Herbert, A., Hoelmer, K., Hooks, C. R., Jones, A., Krawczyk, G., Kuhar, T., Martinson, H., Mitchell, W., Nielsen, A. L., Pfeiffer, D. G., Raupp, M. J., Rodriguez-Saona, C., Shearer, P., Shrewsbury, P., Venugopal, P. D., Whalen, J., Wiman, N. G., Leskey, T. C., \& Tooker, J. F. (2014). Biology, Ecology, and Management of Brown Marmorated Stink Bug (Hemiptera: Pentatomidae). Journal of Integrated Pest Management, 5, 1-13. doi:10.1603/IPM14002.

Rondoni, G., Bertoldi, V., Malek, R., Foti, M. C., Peri, E., Maistrello, L., Haye, T., \& Conti, E. (2017). Native egg parasitoids recorded from the invasive Halyomorpha halys successfully exploit volatiles emitted by the plant-herbivore complex. Journal of Pest Science, 90, 1087-1095.

Schlaepfer, M. A., Runge, M. C., \& Sherman, P. W. (2002). Ecological and evolutionary traps. Trends in Ecology and Evolution, 17, 474-480.

Schlaepfer, M. A., Sherman, P. W., Blossey, B., \& Runge, M. C. (2005). Introduced species as evolutionary traps. Ecology Letters, 8, 241-246.

Sherratt, T. N., \& Mesterton-Gibbons, M. (2013). Models of group or multi-party contests. In I. C. W. Hardy, \& M. Briffa (Eds.), Animal Contests (pp. 33-46). Cambridge: Cambridge University Press.

Sigmund, K. (2010). The Calculus of Selfishness. Princeton, New Jersey: Princeton University Press.

Strogatz, S. H. (2014). Nonlinear Dynamics and Chaos. (2nd ed.). Boulder, Colorado: Westview Press.

Taylor, P. D., \& Jonker, L. (1978). Evolutionarily stable strategies and game dynamics. Mathematical Biosciences, 40, 145-156.

Visser, M. E. (1994). The importance of being large: the relationship between size and fitness in females of the parasitoid Aphaereta minuta (Hymenoptera: Braconidae). Journal of Animal Ecology, 63, 963-978.

\section{Appendix A. Fitness calculation}

We begin by computing a focal $C$-strategist's expected number of offspring from a suitable host, conditional on discovery at time $T$; for a $C$-strategist, suitable host means 
natural host. We denote this conditional payoff by $w_{1}(T)$. With probability $Z(T)$, the host has already been discovered by another individual. With probability $x_{1}$, the first discoverer is another $C$-strategist. In that case, there ensues a contest between equals, which the intruding $C$-strategist wins with probability $q_{I}$, defined by (3). With probability $x_{2}$, the first discoverer is a $D$-strategist, and so the host is unguarded. With probability $x_{3}$, the first discoverer is a $U$-strategist. It has larger size if it emerged from a more deadly host, which happens with probability $\omega_{L}$, defined by (1), and then the intruding $C$-strategist wins with only the reduced probability $q_{I N}$, defined by (4); whereas if the first discoverer emerged from a natural host, which happens with probability $\omega_{N}$, then its probability of victory is $q_{I}$, the same as for a $C$-strategist.

From above, the probability that the host is guarded at time $T$ is $Z(T) \cdot\left(x_{1}+x_{3}\right)$. The host is unguarded if either the first discoverer is a $D$-strategist or the host has not been discovered by time $T$, that is, with probability $Z(T) \cdot x_{2}+1-Z(T)$. In that case, the focal $C$-strategist becomes the owner. With (conditional) probability $1-Y(T)$, the host is not subsequently discovered, the protagonist remains the owner, and its payoff is therefore 1 (unit of fitness). With probability $Y(T)$, however, the $C$-strategist is subsequently intruded upon. Conditional thereon, with probability $x_{2}$ the intruder is a $D$-strategist and the focal $C$-strategist remains the owner, because the host is a natural one. But a contest ensues if the intruder is either a $C$ - or a $U$-strategist. The focal $C$-strategist wins this contest with probability $q_{O}$ if the intruder is normal, which happens if the intruder is a $C$ strategist or with probability $\omega_{N}$ if the intruder is a $U$-strategist, and with probability $q_{O N}$ if the intruder is large, which happens with probility $\omega_{L}$ if the intruder is a $U$-strategist. Thus the payoff from a host located at time $T$ is

$$
\begin{gathered}
w_{1}(T)=Z(T) \cdot\left\{x_{1} \cdot q_{I}+x_{3}\left(\omega_{L} \cdot q_{I N}+\omega_{N} \cdot q_{I}\right)\right\}+ \\
\left\{Z(T) \cdot x_{2}+1-Z(T)\right\} \cdot\{\{1-Y(T)\} \cdot 1+ \\
\left.Y(T)\left\{x_{1} \cdot q_{O}+x_{2} \cdot 1+x_{3}\left(\omega_{L} \cdot q_{O N}+\omega_{N} \cdot q_{O}\right)\right\}\right\},
\end{gathered}
$$

implying

$$
\begin{aligned}
& w_{1}(T)=k+\left\{x_{1} \cdot q_{I}+x_{3}\left(\omega_{L} \cdot q_{I N}+\omega_{N} \cdot q_{I}\right)\right\} Z(T)+x_{2} Z(T)\{1-Y(T)\}+ \\
& +\left\{x_{1} \cdot q_{O}+x_{2}+x_{3}\left(\omega_{L} \cdot q_{O N}+\omega_{N} \cdot q_{O}\right)\right\}\left\{x_{2} Z(T) Y(T)+\{1-Z(T)\} Y(T)\right\}
\end{aligned}
$$

on using (6). But $T$ is a random variable, and so we compute the expected value of $w_{1}(T)$ 
over the distribution of $T$ to obtain the fitness $W_{1}$ to a $C$-strategist:

$$
\begin{aligned}
& W_{1}=\mathrm{E}\left[w_{1}(T)\right] \\
& =k+\left\{x_{1} \cdot q_{I}+x_{3}\left(\omega_{L} \cdot q_{I N}+\omega_{N} \cdot q_{I}\right)\right\} \mathrm{E}[Z(T)]+x_{2} \mathrm{E}[Z(T)\{1-Y(T)\}]+ \\
& \quad\left\{x_{1} \cdot q_{O}+x_{2}+x_{3}\left(\omega_{L} \cdot q_{O N}+\omega_{N} \cdot q_{O}\right)\right\}\left\{x_{2} \mathrm{E}[Z(T) Y(T)]+\mathrm{E}[\{1-Z(T)\} Y(T)]\right\}
\end{aligned}
$$

from (7), where E denotes expected value. But from (8) we obtain

$$
\begin{aligned}
\mathrm{E}[Z(T)] & =1-\gamma \\
\mathrm{E}[Y(T)] & =\int_{0}^{p} \frac{1}{p} Y(t) d t=1+\frac{1-k}{\ln (k)}=1-\gamma \\
\mathrm{E}[Z(T)\{1-Y(T\}] & =\int_{0}^{p} \frac{1}{p} Z(t)\left\{1-Y(t\} d t=\frac{k-1}{\ln (k)}-k=\gamma-k\right. \\
\mathrm{E}[\{1-Z(T\} Y(T)] & =\int_{0}^{p} \frac{1}{p} Y(t)\left\{1-Z(t\} d t=\frac{k-1}{\ln (k)}-k=\gamma-k\right. \\
\mathrm{E}[Z(T) Y(T\}] & =\int_{0}^{p} \frac{1}{p} Z(t) Y(t) d t=1+k+\frac{2(1-k)}{\ln (k)}=1-2 \gamma+k .
\end{aligned}
$$

Note that $0<k<1$ ensures $0<k<\gamma<\frac{1}{2}(1+k)<1$ with

$$
\lim _{k \rightarrow 0} \gamma=0, \quad \lim _{k \rightarrow 1} \gamma=1
$$

so that all expressions in (A.4) are positive. Substituting from (A.4) into (A.3), the fitness to a $C$-strategist is given by

$$
\begin{array}{r}
W_{1}=k+\left\{x_{1} \cdot q_{I}+x_{3}\left(\omega_{L} \cdot q_{I N}+\omega_{N} \cdot q_{I}\right)\right\}(1-\gamma)+x_{2}(\gamma-k)+ \\
\left\{x_{1} \cdot q_{O}+x_{2}+x_{3}\left(\omega_{L} \cdot q_{O N}+\omega_{N} \cdot q_{O}\right)\right\}\left\{x_{2}(1-2 \gamma+k)+(\gamma-k)\right\},
\end{array}
$$

which is a function of the strategy proportions and five dimensionless parameters, namely, $\alpha, \theta, \lambda, \rho$ and $k$.

Next we compute a focal $D$-strategist's expected number of offspring from a suitable host, conditional on discovery at time $T$; for a $D$-strategist, suitable host means a more deadly host yielding fitness $\alpha$. We denote this conditional payoff by $w_{2}(T)$. With probability $Z(T)$, the host has already been discovered by another individual. With probability $x_{1}$, the first discoverer is a $C$-strategist, and so the host is unguarded. With probability $x_{2}$, the first discoverer is another $D$-strategist. In that case, there ensues a contest between equals, which the intruding $D$-strategist wins with probability $q_{I}$, defined by (3). With probability $x_{3}$, the first discoverer is a $U$-strategist. It is large if it emerged from a more deadly host, which happens with probability $\omega_{L}$, defined by (1), and then the intruding $D$-strategist again wins with probability $q_{I}$; whereas if the first discoverer emerged from 
a natural host, which happens with probability $\omega_{N}$, then the intruding $D$-strategist wins with the larger probability $q_{I L}$ defined by (5).

From above, the probability that the host is guarded at time $T$ is $Z(T) \cdot\left(x_{2}+x_{3}\right)$. The host is unguarded if either the first discoverer is a $C$-strategist or the host has not been discovered by time $T$, that is, with probability $Z(T) \cdot x_{1}+1-Z(T)$. In that case, the focal $D$-strategist becomes the owner. With (conditional) probability $1-Y(T)$, the host is not subsequently discovered, the protagonist remains the owner, and its payoff is therefore $\alpha$. With probability $Y(T)$, however, the $D$-strategist is subsequently intruded upon. Conditional thereon, with probability $x_{1}$ the intruder is a $C$-strategist and the focal $D$-strategist remains the owner, because the host is not a natural one. But a contest ensues if the intruder is either a $D$ - or a $U$-strategist. The focal $D$-strategist wins this contest with probability $q_{O}$ if the intruder is large, which happens if the intruder is a $D$ strategist or with probability $\omega_{L}$ if the intruder is a $U$-strategist, and with probability $q_{O N}$ if the intruder is normal, which happens with probility $\omega_{N}$ if the intruder is a $U$-strategist. Thus the payoff from a host located at time $T$ is

$$
\begin{gathered}
w_{2}(T)=Z(T) \cdot\left\{x_{2} \cdot q_{I}+x_{3}\left(\omega_{L} \cdot q_{I}+\omega_{N} \cdot q_{I L}\right)\right\} \alpha+ \\
\left\{Z(T) \cdot x_{1}+1-Z(T)\right\} \cdot\{\{1-Y(T)\} \cdot 1+ \\
\left.Y(T)\left\{x_{1} \cdot 1+x_{2} \cdot q_{O}+x_{3}\left(\omega_{L} \cdot q_{O}+\omega_{N} \cdot q_{O L}\right)\right\}\right\} \alpha,
\end{gathered}
$$

implying

$$
\begin{aligned}
& w_{2}(T)=\left(k+\left\{x_{2} \cdot q_{I}+x_{3}\left(\omega_{L} \cdot q_{I}+\omega_{N} \cdot q_{I L}\right)\right\} Z(T)+x_{1} Z(T)\{1-Y(T)\}+\right. \\
& \left.\quad+\left\{x_{1}+x_{2} \cdot q_{O}+x_{3}\left(\omega_{L} \cdot q_{O}+\omega_{N} \cdot q_{O L}\right)\right\}\left\{x_{1} Z(T) Y(T)+\{1-Z(T)\} Y(T)\right\}\right) \alpha
\end{aligned}
$$

on using (6). But $T$ is a random variable, and so we compute the expected value of $w_{1}(T)$ over the distribution of $T$ to obtain the fitness $W_{2}$ to a $D$-strategist; on using (A.4), we obtain

$$
\begin{aligned}
W_{2}= & \mathrm{E}\left[w_{2}(T)\right] \\
= & \left(k+\left\{x_{2} \cdot q_{I}+x_{3}\left(\omega_{L} \cdot q_{I}+\omega_{N} \cdot q_{I L}\right)\right\}(1-\gamma)+x_{1}(\gamma-k)+\right. \\
& \left.+\left\{x_{1}+x_{2} \cdot q_{O}+x_{3}\left(\omega_{L} \cdot q_{O}+\omega_{N} \cdot q_{O L}\right)\right\}\left\{x_{1}(1-2 \gamma+k)+(\gamma-k)\right\}\right) \alpha,
\end{aligned}
$$

which is again a function of the strategy proportions and five dimensionless parameters, namely, $\alpha, \theta, \lambda, \rho$ and $k$.

Lastly we compute a focal $U$-strategist's expected number of offspring from a suitable host, conditional on discovery at time $T$; for a $U$-strategist, a suitable host means any host. 
We denote this conditional payoff by $w_{3}(T)$. We first compute a focal $U$-strategist's expected number of offspring from a more deadly host, calling this payoff $w_{3 L}(T)$; then we compute a focal $U$-strategist's expected number of offspring from a natural host, calling this payoff $w_{3 N}(T)$. Then because more deadly and natural hosts are found in proportions $\theta$ and $1-\theta$, respectively, we compute $w_{3}(T)$ as

$$
w_{3}(T)=\theta w_{3 L}(T)+(1-\theta) w_{3 N}(T) .
$$

So we first assume that the suitable host is more deadly host. With probability $Z(T)$, this host has already been discovered by another individual. With probability $x_{1}$, the first discoverer is a $C$-strategist, and so the host is unguarded. With probability $x_{2}$, the first discoverer is a $D$-strategist. In that case, there ensues a contest, which the intruding $U$ strategist wins with probability $q_{I}$ if large and $q_{I N}$ if normal. With probability $x_{3}$, the first discoverer is a $U$-strategist, and the intruding $U$-strategist wins the ensuing contest with probability $\omega_{L} \cdot q_{I}+\omega_{N} \cdot q_{I N}$ if the first discoverer is large but with probability $\omega_{L} \cdot q_{I L}+\omega_{N} \cdot q_{I}$ if the first discoverer is normal; that is, the intruding $U$-strategist wins with probability

$$
\begin{aligned}
q_{A} & =\omega_{L} \cdot\left(\omega_{L} q_{I}+\omega_{N} q_{I N}\right)+\omega_{N} \cdot\left(\omega_{L} q_{I L}+\omega_{N} q_{I}\right) \\
& =q_{I}\left\{\omega_{L}^{2}+\omega_{N}^{2}\right\}+\left\{q_{I L}+q_{I N}\right\} \omega_{L} \omega_{N} .
\end{aligned}
$$

From above, the probability that the more deadly host is guarded at time $T$ is $Z(T)$. $\left(x_{2}+x_{3}\right)$. The host is unguarded if either the first discoverer is a $C$-strategist or the host has not been discovered by time $T$, that is, with probability $Z(T) \cdot x_{1}+1-Z(T)$. In that case, the focal $U$-strategist becomes the owner. With (conditional) probability $1-Y(T)$, the host is not subsequently discovered, the protagonist remains the owner, and its payoff is therefore $\alpha$. With probability $Y(T)$, however, the $U$-strategist is subsequently intruded upon. Conditional thereon, with probability $x_{1}$ the intruder is a $C$-strategist and the focal $U$-strategist remains the owner, because the host is not a natural one. But a contest ensues if the intruder is either a $D$ - or a $U$-strategist. If the intruder is a $D$-strategist, then the focal $U$-strategist wins with probability $q_{O}$ if large and $q_{O N}$ if normal, i.e., with probability $\omega_{L} \cdot q_{O}+\omega_{N} \cdot q_{O N}$. If the intruder is a $U$-strategist, then by analogy with (A.11), the focal $U$-strategist wins the contest with probability

$$
\begin{aligned}
q_{B} & =\omega_{L} \cdot\left(\omega_{L} q_{O}+\omega_{N} q_{O L}\right)+\omega_{N} \cdot\left(\omega_{L} q_{O N}+\omega_{N} q_{O}\right) \\
& =q_{O}\left\{\omega_{L}{ }^{2}+\omega_{N}{ }^{2}\right\}+\left\{q_{O L}+q_{O N}\right\} \omega_{L} \omega_{N} .
\end{aligned}
$$


Thus the payoff from a more deadly host located at time $T$ is

$$
\begin{array}{r}
w_{3 L}(T)=Z(T) \cdot\left\{x_{2} \cdot\left(\omega_{L} \cdot q_{I}+\omega_{N} \cdot q_{I N}\right)+x_{3} \cdot q_{A}\right\} \alpha+ \\
\left\{Z(T) \cdot x_{1}+1-Z(T)\right\} \cdot\{\{1-Y(T)\} \cdot 1+ \\
\left.Y(T)\left\{x_{1}+x_{2}\left(\omega_{L} q_{O}+\omega_{N} q_{O N}\right)+x_{3} q_{B}\right\}\right\} \alpha,
\end{array}
$$

implying

$$
\begin{aligned}
w_{3 L}(T) & =\left(k+\left\{x_{2} \cdot\left(\omega_{L} \cdot q_{I}+\omega_{N} \cdot q_{I N}\right)+x_{3} \cdot q_{A}\right\} Z(T)+x_{1} Z(T)\{1-Y(T)\}+\right. \\
+ & \left.\left\{x_{1}+x_{2}\left(\omega_{L} q_{O}+\omega_{N} q_{O N}\right)+x_{3} q_{B}\right\}\left\{x_{1} Z(T) Y(T)+\{1-Z(T)\} Y(T)\right\}\right) \alpha
\end{aligned}
$$

on using (6).

We now assume that the suitable host is a natural host. With probability $Z(T)$, this host has already been discovered by another individual. With probability $x_{1}$, the first discoverer is a $C$-strategist. In that case, there ensues a contest, which the intruding $U$ strategist wins with probability $q_{I L}$ if large and $q_{I}$ if normal, that is, with probability $\omega_{L}$. $q_{I L}+\omega_{N} \cdot q_{I}$. With probability $x_{2}$, the first discoverer is a $D$-strategist, and so the host is unguarded. With probability $x_{3}$, the first discoverer is another $U$-strategist, and the intruding $U$-strategist wins the ensuing contest with probability $\omega_{L} \cdot q_{I}+\omega_{N} \cdot q_{I N}$ if the first discoverer is large but with probability $\omega_{L} \cdot q_{I L}+\omega_{N} \cdot q_{I}$ if the first discoverer is normal; that is, the intruding $U$-strategist wins with probability $q_{A}$ defined by (A.11).

From above, the probability that the natural host is guarded at time $T$ is $Z(T) \cdot\left(x_{1}+x_{3}\right)$. The host is unguarded if either the first discoverer is a $D$-strategist or the host has not been discovered by time $T$, that is, with probability $Z(T) \cdot x_{2}+1-Z(T)$. Then the focal $U$-strategist becomes the owner. With (conditional) probability $1-Y(T)$, the host is not subsequently discovered, the protagonist remains the owner, and its payoff is therefore 1. With probability $Y(T)$, however, the $U$-strategist is subsequently intruded upon. Conditional thereon, with probability $x_{2}$ the intruder is a $D$-strategist and the focal $U$-strategist remains the owner, because the host is not a stink bug. But a contest ensues if the intruder is either a $C$ - or a $U$-strategist. If the intruder is a $C$-strategist, then the focal $U$-strategist wins with probability $q_{O L}$ if large and $q_{O}$ if normal, i.e., with probability $\omega_{L} \cdot q_{O L}+\omega_{N} \cdot q_{O}$. If the intruder is a $U$-strategist, then by analogy with (A.12), the focal $U$-strategist wins the contest with probability $q_{B}$. Thus the payoff from a natural host located at time $T$ is

$$
\begin{gathered}
w_{3 N}(T)=Z(T) \cdot\left\{x_{1} \cdot\left(\omega_{L} \cdot q_{I L}+\omega_{N} \cdot q_{I}\right)+x_{3} \cdot q_{A}\right\}+ \\
\left\{Z(T) \cdot x_{2}+1-Z(T)\right\} \cdot\{\{1-Y(T)\} \cdot 1+ \\
\left.Y(T)\left\{x_{1}\left(\omega_{L} \cdot q_{O L}+\omega_{N} \cdot q_{O}\right)+x_{2}+x_{3} q_{B}\right\}\right\},
\end{gathered}
$$


implying

$$
\begin{array}{r}
w_{3 N}(T)=\left(k+\left\{x_{1} \cdot\left(\omega_{L} \cdot q_{I L}+\omega_{N} \cdot q_{I}\right)+x_{3} \cdot q_{A}\right\} Z(T)+x_{2} Z(T)\{1-Y(T)\}+\right. \\
\left.+\left\{x_{1}\left(\omega_{L} \cdot q_{O L}+\omega_{N} \cdot q_{O}\right)+x_{2}+x_{3} q_{B}\right\}\left\{x_{2} Z(T) Y(T)+\{1-Z(T)\} Y(T)\right\}\right)
\end{array}
$$

on using (6). Substitution from (A.14) and (A.16) into (A.10) now yields $W_{3}(T)$. But $T$ is a random variable, and so we compute the expected value of $w_{3}(T)$ over the distribution of $T$ to obtain the fitness $W_{3}$ to a $D$-strategist; on using (A.4), we obtain

$$
\begin{aligned}
W_{3}= & \mathrm{E}\left[w_{3}(T)\right]=\theta \mathrm{E}\left[w_{3 L}(T)\right]+(1-\theta) \mathrm{E}\left[w_{3 N}(T)\right] \\
= & \theta\left(k+\left\{x_{2} \cdot\left(\omega_{L} \cdot q_{I}+\omega_{N} \cdot q_{I N}\right)+x_{3} \cdot q_{A}\right\}(1-\gamma)+x_{1}(\gamma-k)+\right. \\
& \left.\left.x_{1}+x_{2}\left(\omega_{L} q_{O}+\omega_{N} q_{O N}\right)+x_{3} q_{B}\right\}\left\{x_{1}(1-2 \gamma+k)+\gamma-k\right\}\right) \alpha+ \\
& (1-\theta)\left(k+\left\{x_{1} \cdot\left(\omega_{L} \cdot q_{I L}+\omega_{N} \cdot q_{I}\right)+x_{3} \cdot q_{A}\right\}(1-\gamma)+x_{2}(\gamma-k)+\right. \\
& \left.\left\{x_{1}\left(\omega_{L} \cdot q_{O L}+\omega_{N} \cdot q_{O}\right)+x_{2}+x_{3} q_{B}\right\}\left\{x_{2}(1-2 \gamma+k)+\gamma-k\right\}\right)
\end{aligned}
$$

which is again a function of the strategy proportions and five dimensionless parameters, namely, $\alpha, \theta, \lambda, \rho$ and $k$.

\section{Appendix B. Fitnesses in the limit as $a \rightarrow \infty$}

The expressions for the fitnesses simplify greatly in the limit as $a \rightarrow \infty$ and hence $k \rightarrow 0$ in (7), so that also $\gamma \rightarrow 0$ by (8). From (A.6), (A.9) and (A.17), we obtain

$$
\begin{gathered}
W_{1}=x_{1} \cdot q_{I}+x_{3}\left(\omega_{L} \cdot q_{I N}+\omega_{N} \cdot q_{I}\right)+\left\{x_{1} \cdot q_{O}+x_{2}+x_{3}\left(\omega_{L} \cdot q_{O N}+\omega_{N} \cdot q_{O}\right)\right\} x_{2}, \\
W_{2}=\left(x_{2} \cdot q_{I}+x_{3}\left(\omega_{L} \cdot q_{I}+\omega_{N} \cdot q_{I L}\right)+\left\{x_{1}+x_{2} \cdot q_{O}+x_{3}\left(\omega_{L} \cdot q_{O}+\omega_{N} \cdot q_{O L}\right)\right\} x_{1}\right) \alpha
\end{gathered}
$$

and

$$
\begin{aligned}
& W_{3}= \theta\left(x_{2} \cdot\left(\omega_{L} \cdot q_{I}+\omega_{N} \cdot q_{I N}\right)+x_{3} \cdot q_{A}+\right. \\
&\left.\left\{x_{1}+x_{2}\left(\omega_{L} q_{O}+\omega_{N} q_{O N}\right)+x_{3} q_{B}\right\} x_{1}\right) \alpha+ \\
&(1-\theta)\left(x_{1} \cdot\left(\omega_{L} \cdot q_{I L}+\omega_{N} \cdot q_{I}\right)+x_{3} \cdot q_{A}+\right. \\
&\left.\left\{x_{1}\left(\omega_{L} \cdot q_{O L}+\omega_{N} \cdot q_{O}\right)+x_{2}+x_{3} q_{B}\right\} x_{2}\right) .
\end{aligned}
$$

When (12) holds, these expressions further reduce to

$$
\begin{gathered}
W_{1}=\frac{1}{2}\left(1+x_{2}\right)\left(x_{1}+\omega_{N} x_{3}\right)+x_{2}^{2} \\
W_{2}=\left(\frac{1}{2}\left(1+x_{1}\right)\left(x_{2}+\left\{1+\omega_{N}\right\} x_{3}\right)+x_{1}^{2}\right) \alpha
\end{gathered}
$$


and

$$
\begin{aligned}
W_{3}=\theta\left(\frac{1}{2}\left(1+x_{1}\right)\left(x_{3}+\omega_{L} x_{2}\right)+x_{1}^{2}\right) \alpha+ & \\
& (1-\theta)\left(\frac{1}{2}\left(1+x_{2}\right)\left(x_{3}+\left\{1+\omega_{L}\right\} x_{1}\right)+x_{2}^{2}\right)
\end{aligned}
$$

because $\omega_{L}+\omega_{N}=1$ by (1), and so (12) implies $q_{A}=q_{B}=\frac{1}{2}\left(\omega_{L}+\omega_{N}\right)^{2}=\frac{1}{2}$ by (A.11) and (A.12).

\section{Appendix C. The strategy mix without $U$-strategists for $k \rightarrow \infty, \rho=0, \lambda \rightarrow \infty$}

When $x_{3}=0$, the third equation in (9) becomes an identity; the second becomes superfluous, because $x_{2}=1-x_{1}$ by (11); and because $W_{1}-\bar{W}=1 \cdot W_{1}-\bar{W}=\left(x_{1}+x_{2}\right) W_{1}-$ $\left\{x_{1} W_{1}+x_{2} W_{2}\right\}=x_{2}\left(W_{1}-W_{2}\right)$, when (12) holds the first equation reduces to

$$
\frac{d x_{1}}{d t}=\frac{1}{2} x_{1} x_{2}\left\{\left(1-x_{1}\right)^{2}-\alpha\left(1+x_{1}^{2}\right)+1\right\} \text {. }
$$

If $\alpha<\frac{1}{2}$, then the term in squiggly brackets is strictly positive, implying $x_{1} \rightarrow 1, x_{2} \rightarrow 0$ as $t \rightarrow \infty$. If $\frac{1}{2}<\alpha<1$, however, it becomes negative for $\frac{1}{2}<x_{1 r}<x_{1}<1$, implying $x_{1} \rightarrow x_{1 r}, x_{2} \rightarrow x_{2 r}$ as $t \rightarrow \infty$ where $x_{1 r}=1-x_{2 r}$ and

$$
x_{2 r}=\frac{\sqrt{\alpha-(1-\alpha)^{2}}-\alpha}{1-\alpha}
$$

(which increases from 0 to $\frac{1}{2}$ as $\alpha$ increases from $\frac{1}{2}$ towards 1 ). We note in passing that $\frac{1}{2}<x_{2 r}<1$ for $1<\alpha<2$ and $H$ goes to fixation for $\alpha>2$ because the term in squiggly brackets in (C.1) becomes strictly negative-in perfect accord with intuition, but also irrelevant, because $\alpha<1$ is an assumption of our analysis. The equilibrium $\left(x_{1 r}, x_{2 r}\right)$ remains an equilibrium when $U$ is introduced to the strategy mix but is no longer an attractor: it becomes an unstable saddle point on the "roof-edge" of $\Delta$, as illustrated by Figure 3(b).

\section{Appendix D. Phase-plane analysis for $k=0, \rho=0, \lambda \rightarrow \infty$}

The phase plane is analyzed by standard techniques of nonlinear analysis (e.g., Strogatz, 2014; Layek, 2015). In particular, the equilibrium points are classified as described by Mesterton-Gibbons (2019, p. 93). In general, if $\left(\tilde{x}_{1}, \tilde{x}_{2}\right)$ is an equilibrium point of the dynamical system

$$
\frac{d x_{1}}{d t}=G_{1}\left(x_{1}, x_{2}\right), \quad \frac{d x_{2}}{d t}=G_{2}\left(x_{1}, x_{2}\right),
$$

that is if $G_{1}\left(\tilde{x}_{1}, \tilde{x}_{2}\right)=0=G_{2}\left(\tilde{x}_{1}, \tilde{x}_{2}\right)$, then its type is determined by the eigenvalues of the Jacobian matrix $J\left(\tilde{x}_{1}, \tilde{x}_{2}\right)$ having $g_{i j}\left(\tilde{x}_{1}, \tilde{x}_{2}\right)=\partial G_{i} /\left.\partial x_{j}\right|_{x_{1}=\tilde{x}_{1}, x_{2}=\tilde{x}_{2}}$ in row $i$ and column 
$j$, and hence by the roots-termed $r_{1}\left(\tilde{x}_{1}, \tilde{x}_{2}\right)$ and $r_{2}\left(\tilde{x}_{1}, \tilde{x}_{2}\right)$ - of its characteristic equation $r^{2}-\left\{g_{11}\left(\tilde{x}_{1}, \tilde{x}_{2}\right)+g_{22}\left(\tilde{x}_{1}, \tilde{x}_{2}\right)\right\} r+g_{11}\left(\tilde{x}_{1}, \tilde{x}_{2}\right) g_{22}\left(\tilde{x}_{1}, \tilde{x}_{2}\right)-g_{12}\left(\tilde{x}_{1}, \tilde{x}_{2}\right) g_{21}\left(\tilde{x}_{1}, \tilde{x}_{2}\right)=0$. Those roots are real whenever the discriminant

$$
\delta_{\mathrm{CE}}\left(\tilde{x}_{1}, \tilde{x}_{2}\right)=\left(g_{11}\left(\tilde{x}_{1}, \tilde{x}_{2}\right)-g_{22}\left\{\tilde{x}_{1}, \tilde{x}_{2}\right)\right\}^{2}+4 g_{12}\left(\tilde{x}_{1}, \tilde{x}_{2}\right) g_{21}\left(\tilde{x}_{1}, \tilde{x}_{2}\right)
$$

of the characteristic equation is positive, and in particular when $g_{12}\left(\tilde{x}_{1}, \tilde{x}_{2}\right)$ and $g_{21}\left(\tilde{x}_{1}, \tilde{x}_{2}\right)$ have the same sign (or at least one of them is zero, as at all three vertices of the phase triangle $\Delta$ ). The equilibrium is stable if both roots have a negative real part, and otherwise it is unstable. For real roots, $\left(\tilde{x}_{1}, \tilde{x}_{2}\right)$ is a stable node, an unstable node or a saddle point according to whether the eigenvalues are both negative, both positive or have opposite signs; and for complex conjugate roots, $\left(\tilde{x}_{1}, \tilde{x}_{2}\right)$ is an unstable or stable focus, according to whether the eigenvalues have a positive or negative real part. We note in passing that these classification criteria do not apply to non-hyperbolic equilibrium points at which the real part of an eigenvalue is zero, but they suffice for our purposes because we make the generic payoffs assumption (Broom \& Rychtář, 2013, p. 21; Mesterton-Gibbons, 2019, p. 13), and non-hyperbolic equilibria are non-generic.

We now proceed with identifying all equilibria. It is clear from inspection of (15) that $(0,0),(1,0)$ and $(0,1)$ are all equilibrium points because $\phi_{1}(1,0)=\phi_{2}(0,1)=0$. On setting $G_{1}\left(x_{1}, x_{2}\right)=x_{1} \phi_{1}\left(x_{1}, x_{2}\right)$ and $G_{2}\left(x_{1}, x_{2}\right)=x_{2} \phi_{2}\left(x_{1}, x_{2}\right)$ in (D.1), we find that the eigenvalues at $(0,0)$ are

$$
r_{1}(0,0)=-\frac{1}{2} \omega_{N} \sigma_{0 b}(\alpha, \theta), \quad r_{2}(0,0)=\frac{1}{2 \alpha} \omega_{L} \sigma_{0 b}(\alpha, \theta)
$$

where $\sigma_{0 b}$ is defined by (D.8) below. Because they have opposite signs, $(0,0)$ is invariably a saddle point. Similarly, the eigenvalues at $(0,1)$ are

$$
r_{1}(0,1)=\frac{1}{2}(2-\alpha), \quad r_{2}(0,1)=\frac{1}{2}(1-\theta)\left\{(1-\alpha) \omega_{L}+(2-\alpha) \omega_{N}\right\} .
$$

Both are positive, hence $(0,1)$ is invariably an unstable node. The eigenvalues at $(1,0)$ are

$$
r_{1}(1,0)=-\frac{1}{2}(1-2 \alpha), \quad r_{2}(1,0)=-\frac{1}{2 \alpha} \omega_{L} \sigma_{1 b}(\alpha, \theta) .
$$

The first eigenvalue is negative for $\alpha<\frac{1}{2}$ and positive for $\alpha>\frac{1}{2}$. The second eigenvalue is negative to the left of the curve separating region iv $\cup v$ from region $i \cup$ ii $\cup$ iii $\cup$ vi in Figure 1 , and $r_{2}>0$ to the right of this curve. Thus $(1,0)$ is a stable node when $(\alpha, \theta)$ lies in region iv $\cup \mathrm{v}$, a saddle point when $(\alpha, \theta)$ lies in region $\mathrm{ii} \cup$ iii $\cup$ vi and an unstable node when $(\alpha, \theta)$ lies in region i. We note in passing that $(1,0)$ is non-hyperbolic where $\sigma_{1 b}=0$ or $\alpha=\frac{1}{2}$; these points form a set of measure zero, and hence are non-generic. Such a nonhyperbolic equilibrium is exemplified by $(1,0)$ in Figure 4 , for which nonlinear terms in 
the Taylor expansion of (15) about this point can be used to classify it as a saddle-node equilibrium (Layek, 2015); however, trajectories in its vicinity still resemble those near a saddle point, as Figure 4 illustrates.

A "roof-edge" equilibrium $\left(x_{1 r}, x_{2 r}\right)$ on the hypotenuse of $\Delta$ has already been identified in Appendix C; it exists as long as $\frac{1}{2}<\alpha<1$, that is, when $(\alpha, \theta)$ lies in region $\mathrm{i}$ of Figure 1, as illustrated by Figure 3(b). Because $r_{1}\left(x_{1 r}, x_{2 r}\right)$ and $r_{2}\left(x_{1 r}, x_{2 r}\right)$ are the roots of a quadratic equation, explicit expressions for them, their product $r_{1}\left(x_{1 r}, x_{2 r}\right) r_{2}\left(x_{1 r}, x_{2 r}\right)$ and the discriminant $\delta_{\mathrm{CE}}\left(x_{1 r}, x_{2 r}\right)$ are easily determined. They are continuous functions of $\alpha$ and $\theta$, but they are too cumbersome for useful analysis. A much more efficient way to determine their signs inside a region is to use constrained numerical optimization (e.g., Bertsekas, 2016; Aragón et al., 2019), for which powerful and reliable mathematical packages are now widely available. If a discriminant $\delta_{\mathrm{CE}}\left(\tilde{x}_{1}, \tilde{x}_{2}\right)$ has a minimum of zero over some region, then the associated eigenvalues must be real on that region. If, further, the eigenvalue product $r_{1}\left(\tilde{x}_{1}, \tilde{x}_{2}\right) r_{2}\left(\tilde{x}_{1}, \tilde{x}_{2}\right)$ has a maximum of 0 on the boundary of the region, then it must be negative inside the region, and so $\left(\tilde{x}_{1}, \tilde{x}_{2}\right)$ must be a saddle point; whereas if both the product $r_{1}\left(\tilde{x}_{1}, \tilde{x}_{2}\right) r_{2}\left(\tilde{x}_{1}, \tilde{x}_{2}\right)$ has a minimum of zero on the boundary and the sum $r_{1}\left(\tilde{x}_{1}, \tilde{x}_{2}\right)+r_{2}\left(\tilde{x}_{1}, \tilde{x}_{2}\right)$ has either a maximum of zero or a minimum of zero on the boundary, then $\left(\tilde{x}_{1}, \tilde{x}_{2}\right)$ must be either a stable or an unstable node, respectively. The requisite properties can all be readily established by numerical optimization.

In the case of $\left(x_{1 r}, x_{2 r}\right)$, over region $\mathrm{i}$ in Figure 1 we find that $\delta_{\mathrm{CE}}\left(x_{1 r}, x_{2 r}\right)$ has a minimum of 0 at both $\left(\frac{1}{2}, 0\right)$ and $\left(\frac{1}{2}, 1\right)$; and that $r_{1}\left(x_{1 r}, x_{2 r}\right) r_{2}\left(x_{1 r}, x_{2 r}\right)$ has a maximum of 0 , which occurs all the way around the boundary for $\theta=0, \alpha \rightarrow 1, \theta=1$ and $\alpha=\frac{1}{2}$. For all $(\alpha, \theta)$ satisfying $\frac{1}{2}<\alpha<1,0<\theta<1$, therefore, $r_{1}\left(x_{1 r}, x_{2 r}\right) r_{2}\left(x_{1 r}, x_{2 r}\right)<0$. Hence $\left(x_{1 r}, x_{2 r}\right)$ is invariably a saddle point, the boundary of region I being excluded by (2).

Let us now turn our attention to the possibility of equilibria on the base edge of $\Delta$ where $0<x_{1}<1, x_{2}=0$ or on the side edge where $x_{1}=0,0<x_{2}<1$. From (15) we find that

$$
\phi_{1}\left(x_{1}, 0\right)=\left(1-x_{1}\right) Q_{1}\left(x_{1}\right), \quad \phi_{2}\left(0, x_{2}\right)=\left(1-x_{2}\right) Q_{2}\left(x_{2}\right)
$$

where $Q_{1}$ and $Q_{2}$ are quadratic polynomials defined by

$$
\begin{aligned}
& Q_{1}\left(x_{1}\right)=\frac{1}{2}\left\{\theta\left\{1-\alpha\left(1+x_{1}^{2}\right)+\omega_{L} x_{1}\right\}-\omega_{L}\right\} \\
& Q_{2}\left(x_{2}\right)=\frac{1}{2}\left\{\left(2-\theta-\omega_{L}\right) \alpha-(1-\theta)\left\{1+x_{2}^{2}+\left(1-\omega_{L}\right) \alpha x_{2}\right\}\right\}
\end{aligned}
$$

with $\omega_{L}$ defined by (1). A base-edge equilibrium occurs where $Q_{1}\left(x_{1}\right)=0$, and a side-edge equilibrium occurs where $Q_{2}\left(x_{2}\right)=0$. We deal with base-edge equilibria first.

In that regard, the signs of $Q_{1}(0)$ and $Q_{1}(1)$ are determined by $\sigma_{0 b}$ and $\sigma_{1 b}$, respectively, 
where we define

$$
\sigma_{0 b}(\alpha, \theta)=(1-\theta)(1-2 \alpha)-\alpha^{2} \theta, \quad \sigma_{1 b}(\alpha, \theta)=2 \alpha \theta(2-\alpha)-3 \alpha-\theta+1 .
$$

The quadratic equation $Q_{1}\left(x_{1}\right)=0$ has two real roots whenever the discriminant $\delta_{1 b}$ is positive, where we define

$$
\delta_{b}(\alpha, \theta)=\alpha^{2} \theta^{4}+4 \alpha \theta^{2}(1-\theta+\alpha \theta) \sigma_{0 b}(\alpha, \theta) .
$$

In Figure 1, $\sigma_{0 b}$ is positive in region $\mathrm{v} \cup$ vi and negative in region $\mathrm{i} \cup$ ii $\cup$ iii $\cup$ iv, whereas $\sigma_{1 b}$ is positive in region iv $\cup \mathrm{v}$ and negative in region $\mathrm{i} \cup \mathrm{ii} \cup \mathrm{iii} \cup \mathrm{vi}$; and $\delta_{b}$ is positive in region iii $\cup \mathrm{iv} \cup \mathrm{v} \cup$ vi but negative in region $\mathrm{i} \cup$ ii. Thus, because $Q_{1}\left(x_{1}\right)=0$ implies $x_{1}<0$ or $x_{1}>1$ if $Q_{1}(0)$ and $Q_{1}(1)$ are both positive and $\delta_{b}<0$ implies $Q\left(x_{1}\right)<0$ for all $x_{1}$, no point either in $\mathrm{i} \cup \mathrm{ii}$ or in region $\mathrm{v}$ of Figure 1 corresponds to a base-edge equilibrium. Any point in region iv $\cup$ vi corresponds to a unique base-edge equilibrium because $Q_{1}(0)$ and $Q_{1}(1)$ have opposite signs: $Q_{1}(0)<0, Q_{1}(1)>0$ in region iv, whereas $Q_{1}(0)>0, Q_{1}(1)<0$ in region vi. In region iii, however, there are always two base-edge equilibria because the whole of region iii lies below the curve from $\left.\frac{1}{2}, 1\right)$ to $\left(0, \frac{2}{3}\right)$ with equation $\theta=2 /\{3-2 \alpha\}$ (not shown in Figure 1), implying that $\omega_{L}<2 \alpha$ and hence that $\partial Q_{1} /\left.\partial x_{2}\right|_{x_{2}=0}=\frac{1}{2} \theta \omega_{L}>0$ and $\partial Q_{1} /\left.\partial x_{2}\right|_{x_{2}=1}=\frac{1}{2} \theta\left(\omega_{L}-2 \alpha\right)<0$ have opposite signs, and so the positive maximum of $Q_{1}$ must occur within $(0,1)$. The dot indicates such a point in region iii, corresponding to two base-edge equilibria. Here $\alpha=0.42$ and $\theta=0.7$, implying $\sigma_{0 b}(\alpha, \theta)=-0.7548 \times 10^{-1}$, $\sigma_{1 b}(\alpha, \theta)=-0.3096 \times 10^{-1}$ and $\delta_{b}(\alpha, \theta)=0.5445 \times 10^{-2}$. The corresponding equilibria are a saddle point at $(0.3779,0)$ and a globally attracting stable node at $(0.8005,0)$, as illustrated by Figure 2(b).

If $\left(x_{1 b}, 0\right)$ denotes a base-edge equilibrium, then $Q_{1}\left(x_{1}\right)=0$ implies

$$
x_{1 b}^{ \pm}=\frac{\omega_{L} \pm \sqrt{4 \alpha\left(1-\alpha-\omega_{L} / \theta\right)+\omega_{L}^{2}}}{2 \alpha}
$$

by (D.7), where the square-root sign is positive for the base-edge equilibrium that exists throughout region iii $\cup$ iv $\cup v i$ and negative for the additional equilibrium that exists only in region iii. As before, because $r_{1}\left(x_{1 b}^{ \pm}, 0\right)$ and $r_{2}\left(x_{1 b}^{ \pm}, 0\right)$ are the roots of a quadratic equation, explicit expressions for them, their product $r_{1}\left(x_{1 b}^{ \pm}, 0\right) r_{2}\left(x_{1 b}^{ \pm}, 0\right)$ and $\delta_{\mathrm{CE}}\left(x_{1 b}^{ \pm}, 0\right)$ are easily determined as (extremely cumbersome) continuous functions of $\alpha$ and $\theta$ on using (1). The minimum value of $\delta_{\mathrm{CE}}\left(x_{1 b}^{+}, 0\right)$ over region iv of Figure 1 is 0 at $(1 / 2,1)$, so the eigenvalues are real. The maximum value of $r_{1}\left(x_{1 b}^{+}, 0\right) r_{2}\left(x_{1 b}^{+}, 0\right)$ over region IV is 0 ; it occurs along the boundary with region iii between $\left(\alpha_{c}, \theta_{c}\right)$ and $\left(\frac{1}{2}, 1\right)$, and along the upper edge between $\left(\frac{1}{2}, 1\right)$ and $(0,1)$. For all $(\alpha, \theta)$ in the interior of region iv, however, $r_{1}\left(x_{1 b}^{+}, 0\right) r_{2}\left(x_{1 b}^{+}, 0\right)<0$, 
and so $\left(x_{1 b}^{+}, 0\right)$ is always a saddle point in region iv. The eigenvalues are likewise real on region vi of Figure 1, since the minimum value of $\delta_{\mathrm{CE}}\left(x_{1 b}^{+}, 0\right)$ over region vi is 0 at $(1 / 2,0)$. The minimum value of $r_{1}\left(x_{1 b}^{+}, 0\right) r_{2}\left(x_{1 b}^{+}, 0\right)$ over region vi is 0 ; it occurs along the boundary with region $\mathrm{v}$ between $\left(\alpha_{c}, \theta_{c}\right)$ and $\left(\frac{1}{3}, 0\right)$, and along the lower edge between $\left(\frac{1}{3}, 0\right)$ and $\left(\frac{1}{2}, 0\right)$. For all $(\alpha, \theta)$ in the interior of region vi, however, $r_{1}\left(x_{1 b}^{+}, 0\right) r_{2}\left(x_{1 b}^{+}, 0\right)>0$. Furthermore, the sum of eigenvalues $r_{1}\left(x_{1 b}^{+}, 0\right)+r_{2}\left(x_{1 b}^{+}, 0\right)$ has a maximum over region vi of 0 , uniquely at $\left(\frac{1}{2}, 0\right)$, implying that the sum of eigenvalues is always negative within region vi. So $\left(x_{1 b}^{+}, 0\right)$ is always a stable node in region vi.

Over region iii of Figure 1, the eigenvalues are still real because the minimum value of $\delta_{\mathrm{CE}}\left(x_{1 b}^{+}, 0\right)$ over region iii is still 0 at $(1 / 2,0)$, the same as for region vi. If the negative sign is taken in (D.10), then over region iii the eigenvalue product $r_{1}\left(x_{1 b}^{-}, 0\right) r_{2}\left(x_{1 b}^{-}, 0\right)$ has a maximum of 0 along the boundary from $\left(\alpha_{c}, \theta_{c}\right)$ to $\left(\frac{1}{2}, 0\right)$ that separates region iii from region vi, implying that the additional base-edge equilibrium is always a saddle point. If instead the positive sign is taken in (D.10), then the eigenvalue product $r_{1}\left(x_{1 b}^{+}, 0\right) r_{2}\left(x_{1 b}^{+}, 0\right)$ over region iii has a minimum of 0 along the curve from $\left(\frac{1}{2}, 0\right)$ to $\left(\frac{1}{2}, 1\right)$ where $\delta_{b}(\alpha, \theta)=0$ and along the curve from $\left(\frac{1}{2}, 1\right)$ to $\left(\alpha_{c}, \theta_{c}\right)$ where $\sigma_{1 b}(\alpha, \theta)=0$; these two curves separate region ii from region ii and region iv, respectively. Moreover, the eigenvalue sum $r_{1}\left(x_{1 b}^{+}, 0\right)+r_{2}\left(x_{1 b}^{+}, 0\right)$ has a maximum of 0 at $\left(\frac{1}{2}, 0\right)$ and $\left(\frac{1}{2}, 1\right)$; it also has a local maximum of approximately -0.0356 at the point where the curve $\delta_{b}(\alpha, \theta)=0$ separating region ii from region iii has a vertical tangent, which we denote by $\left(\alpha_{*}, \theta_{*}\right)$. Because the eigenvalues have a positive product and negative sum throughout the interior of region iii, we conclude that $\left(x_{1 b}^{+}, 0\right)$ is invariably a stable node. Note that $\alpha_{*}$ is the only zero between 0 and 1 of the cubic equation

$$
\alpha^{3}-2 \alpha^{2}+3 \alpha-1=0 .
$$

Thus $\alpha_{*} \approx 0.4302$ with $\theta_{*} \approx 0.691$.

The second polynomial is more straightforward to deal with. It is easily verified that $Q_{2}(0)$ must have the sign of $\sigma_{0 s}=-\sigma_{0 b}$, that is, the opposite sign from $Q_{1}(0)$; whereas $Q_{2}(1)$ must have the sign of $-\{(2-\alpha)(1-\theta)+(1-\alpha) \alpha \theta\}$, which is always negative. The equation $Q_{2}\left(x_{2}\right)=0$ has two real roots whenever the discriminant $\delta_{s}$ is positive, where

$$
\delta_{s}(\alpha, \theta)=\alpha^{2}(1-\theta)^{2}+4\{1-(1-\alpha) \theta\}\left\{\left(1+\alpha^{2}\right) \theta+2 \alpha(1-\theta)-1\right\} .
$$

So there is a narrow subset of region $\mathrm{v} \cup$ vi in Figure 1(a) to the right of the dashed curve where $Q_{2}(0)<0, Q_{2}(1)<0$ and $\delta_{s}>0$, implying that $Q_{2}\left(x_{2}\right)=0$ has two real solutions. Points within this narrow region do not, however, correspond to a pair of side-edge equilibria because $\partial Q_{2} /\left.\partial x_{2}\right|_{x_{2}=0}=-\frac{1}{2} \alpha(1-\theta)\left(1-\omega_{L}\right)$ and $\partial Q_{2} /\left.\partial x_{2}\right|_{x_{2}=1}=$ $-\frac{1}{2}(1-\theta)\left\{2+\alpha\left(1-\omega_{L}\right)\right\}$ are both negative, implying that $Q_{2}\left(x_{2}\right)=0$ where $x_{2}<-1$. 
Thus no point in region $\mathrm{v} \cup$ vi of Figure 1 corresponds to a side-edge equilibrium. A sideedge equilibrium $\left(0, x_{2 s}\right)$ does exist for every $(\alpha, \theta)$ in region $\mathrm{i} \cup \mathrm{ii} \cup \mathrm{iii} \cup \mathrm{iv}$, and $\delta_{\mathrm{CE}}\left(0, x_{2 s}\right)$ has a minimum of 0 along its boundary with region $\mathrm{v} \cup \mathrm{vi}$, establishing that the eigenvalues are real; but the product of eigenvalues has a maximum of 0 on the same curve, as well on the line joining $(0,1)$ to $(1,1)$ where $\theta=0$. So $r_{1}\left(0, x_{2 s}\right) r_{2}\left(0, x_{2 s}\right)<0$ within region $\mathrm{i} \cup \mathrm{ii} \cup \mathrm{iii} \cup \mathrm{iv}$, implying that $\left(0, x_{2 s}\right)$ is invariably a saddle point. We do not present the explicit expression for $x_{2 s}$ as a function of $\alpha$ and $\theta$ because it is so surprisingly cumbersome.

We have now classified all possible equilibrium points on the boundary of $\Delta$. Any other equilibrium point must lie in its interior and therefore satisfy $\phi_{1}\left(x_{1}, x_{2}\right)=0=$ $\phi_{2}\left(x_{1}, x_{2}\right)$. We have not been able to establish analytically that $\phi_{1}\left(x_{1}, x_{2}\right)=0=\phi_{2}\left(x_{1}, x_{2}\right)$ and $\left(x_{1}, x_{2}\right) \in \Delta$ imply $x_{1}=x_{2}$ when $\rho=0$, but an exhaustive numerical search of the parameter square $S$ reveals that all interior equilibrium points do in fact lie on the open line segment $\Lambda$ defined by (17). Let $(p, p) \in \Lambda$ be such an equilibrium point, and define

$$
Q(p)=\left\{\sigma_{0 b}+3 \alpha\right\} p^{2}+\alpha p+\sigma_{0 b}
$$

where $\sigma_{0 b}$ is defined by (D.8). Then

$$
\begin{aligned}
2(1-\theta+\alpha \theta) \phi_{1}(p, p) & =\{p+\theta(1-2 p)\} Q(p) \\
\left.2(1-\theta+\alpha \theta) \phi_{2}(p, p)\right) & =-\{(1-\theta)(1-p)+\theta p\} Q(p),
\end{aligned}
$$

and because $(p, p) \in \Lambda$ guarantees that $p+\theta(1-2 p)$ and $(1-\theta)(1-p)+\theta p$ are both positive, it follows from $\phi_{1}(p, p)=0=\phi_{2}(p, p)$ that $Q(p)=0$.

Note that $Q\left(\frac{1}{2}\right)=\frac{5}{4}(1-\alpha)(1-\theta+\alpha \theta), \partial Q /\left.\partial p\right|_{p=\frac{1}{2}}=\{2+(2-\alpha) \theta\} \alpha+1-\theta$ and $\partial Q /\left.\partial p\right|_{p=0}=\alpha$ are all positive. If also $Q(0)>0$, that is, if $(\alpha, \theta)$ lies in region III of Figure $1(\mathrm{~b})$, then $Q(p)>0$ for all $0 \leq p \leq \frac{1}{2}$ and there is no interior equilibrium. Elsewhere, however, that is for $(\alpha, \theta)$ in region I $\cup$ II of Figure $1(\mathrm{~b}), Q(0)<0<Q\left(\frac{1}{2}\right)$ implies that an interior equilibrium $\left(p_{*}, p_{*}\right) \in \Lambda$ must exist, where

$$
p_{*}=\frac{-2 \sigma_{0 b}}{\sqrt{\alpha^{2}-4 \sigma_{0 b}\left\{\sigma_{0 b}+3 \alpha\right\}}+\alpha}
$$

with $\sigma_{0 b}$ defined by (D.8). Note that the term inside the square root sign must be positive, because $\sigma_{0 b}$ is negative for all $(\alpha, \theta)$ in region IUII while $\sigma_{0 b}+3 \alpha$ is positive for all $(\alpha, \theta) \in S$.

As before, an explicit expression for $\delta_{\mathrm{CE}}\left(p_{*}, p_{*}\right)$ as a continuous function of $\alpha$ and $\theta$ is straightforward to obtain, but it is far too unwieldy for its presentation to serve any useful purpose. The function assumes the value zero on the closed curve shown in Figure 1(b). Inside the curve, throughout region II, $\delta_{\mathrm{CE}}\left(p_{*}, p_{*}\right)<0$ implies that the eigenvalues are complex, and so $\left(p_{*}, p_{*}\right)$ must be a focus. Outside the curve, in both (disconnected) 
subregions of region I, $\delta_{\mathrm{CE}}\left(p_{*}, p_{*}\right)>0$ implies that the eigenvalues are real. But the eigenvalue product $r_{1}\left(p_{*}, p_{*}\right) r_{2}\left(p_{*}, p_{*}\right)$ has a minimum over region I $\cup$ II in Figure 1(b) of zero, all the way along the boundary between $(1 / 2,0)$ to $(0,1)$ that separates region I $\cup$ II from region III. So $r_{1}\left(p_{*}, p_{*}\right) r_{2}\left(p_{*}, p_{*}\right)$ is never negative, implying that an interior equilibrium is never a saddle point. Hence $\left(p_{*}, p_{*}\right)$ is a node in region I and a focus in region II.

The eigenvalue sum $r_{1}\left(p_{*}, p_{*}\right)+r_{2}\left(p_{*}, p_{*}\right)$ assumes the value zero along the curve in Figure $1(\mathrm{~b})$ that extends from the point $\left(\alpha_{2}, 0\right) \approx(0.628,0)$ where region II touches the line $\theta=1$ to the point $\left(\sqrt{2}-1, \frac{1}{2}\right) \approx(0.414,0)$ where region II touches region III. Below this curve, $r_{1}\left(p_{*}, p_{*}\right)+r_{2}\left(p_{*}, p_{*}\right)$ is negative (with a minimum of $-\frac{1}{4}$ along the line $\alpha=1$ ); above the curve, $r_{1}\left(p_{*}, p_{*}\right)+r_{2}\left(p_{*}, p_{*}\right)$ is positive (with a maximum of about 0.0662 where $\alpha \approx 0.304$ and $\theta=1)$. Hence $\left(p_{*}, p_{*}\right)$ is a stable node in region Ia, a stable focus in region IIa, an unstable focus in region IIb and an unstable node in region IIa.

We have now in effect determined the final destination of $\left(x_{1}(t), x_{2}(t)\right)$ for all $(\alpha, \theta) \in S$. For $(\alpha, \theta)$ in region III of Figure 1(b), the unique local attractor is a stable node that lies at $(1,0)$ in $\Delta$ for $(\alpha, \theta)$ to the left of the dashed curve and at $\left(x_{1 b}, 0\right)$ in $\Delta$ for $(\alpha, \theta)$ to the right of the dashed curve; because this node is the only local attractor, it must also be the global attractor. So the population evolves to either a monomorphism of $C$ or a polymorphism of $C$ and $U$ as indicated in Figure 5.

For region Ia $\cup I$ IIa in Figure $1(b)$, if $(\alpha, \theta)$ lies to the right of the dashed curve from $\left(\frac{1}{2}, 0\right)$ to $\left(\frac{1}{2}, 1\right)$ bounding region iii, the unique local attractor is $\left(p_{*}, p_{*}\right)$, which is a stable node for region Ia and a stable focus for region IIa. A unique local attractor is also the global attractor, so $\left(x_{1}(\infty),\left(x_{2}(\infty)=\left(p_{*}, p_{*}\right)\right.\right.$ for region IIa $\cap \overline{\mathrm{iii}}$, whose phase plane is illustrated by Figure 3(a): the population evolves to a polymorphism of all three strategies. The phase

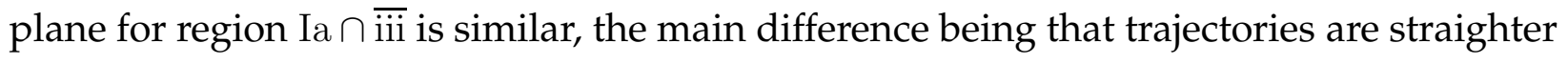
near a node than near a focus (as illustrated by Figure 6(a)). For $(\alpha, \theta)$ on the left of the dashed curve, however, in a narrow wedge sandwiched between the dashed lines converging on $\left(\frac{1}{2}, 0\right)$ and bounded above by the solid curve where $r_{1}\left(p_{*}, p_{*}\right)+r_{2}\left(p_{*}, p_{*}\right)=0$, the base-edge equilibrium $\left(x_{1 b}, 0\right)$ is also a local attractor. Here $\left(x_{1}(0), x_{2}(0)\right)$ determines which local attractor corresponds to $\left(x_{1}(\infty), x_{2}(\infty)\right)$. Regardless of whether $\left(p_{*}, p_{*}\right)$ is a node or a focus, it lies between the origin and a separatrix that joins the unstable node at $(0,1)$ to a saddle point at $\left(x_{1 b}^{-}, 0\right)$. If $\left(x_{1}(0), x_{2}(0)\right)$ lies to the left of this separatrix, then $\left(x_{1}(\infty), x_{2}(\infty)\right)=\left(p_{*}, p_{*}\right)$; if $\left(x_{1}(0), x_{2}(0)\right)$ lies to the right of the separatrix, then $\left(x_{1}(\infty), x_{2}(\infty)\right)=\left(x_{1 b}^{+}, 0\right)$ instead. This outcome is illustrated by Figure $3(\mathrm{~b})$.

We have already established that $(1,0)$ and $\left(x_{1 b}, 0\right)$ are stable nodes in regions iv and iii, respectively; they are also unique local attractors in these regions. They are therefore also globally attracting, that is, $\left(x_{1}(\infty), x_{2}(\infty)\right)=(1,0)$ for $(\alpha, \theta)$ in region iv and 
$\left(x_{1}(\infty), x_{2}(\infty)\right)=\left(x_{1 b}, 0\right)$ for $(\alpha, \theta)$ in region iii, as illustrated by Figure 2(a) and Figure 2(b), respectively. It remains only to determine the final destination of $\left(x_{1}(t), x_{2}(t)\right)$ for $(\alpha, \theta)$ in the intersection of regions $\mathrm{i} \cup \mathrm{i}$ and $\mathrm{Ib} \cup \mathrm{IIb}$. For this subset of $S$, the only equilibrium point-either on the boundary of $\Delta$ or in its interior-is an unstable node (in a tiny subset of region Ib) or an unstable focus (in a much larger subset of region IIb), and $\left(x_{1}(t), x_{2}(t)\right)$ approaches a limit cycle surrounding the unstable source at $\left(p_{*}, p_{*}\right)$ as $t \rightarrow \infty$. Strategy $D$ persists, but within a periodic polymorphism of all three strategies, as opposed to one in which the three proportions are fixed. For illustration, see Figure 4.

\section{Appendix E. Effects of allowing $k, \lambda$ and $\rho$ to depart from their reduced-model values}

In $\S 3$ we focused on our reduced model by assuming extreme parasitoid density ( $a \rightarrow$ $\infty$ or $k=0)$, perfectly decisive size advantage $(\lambda \rightarrow \infty)$ and no owner advantage $(\rho=0)$. In $\S 4$ we considered effects of relaxing those assumptions by allowing the relevant parameters to depart from their limiting values in $\S 3$, but we provided few details, especially concerning the effect of increasing $k$ or decreasing $\lambda$, so that we could focus on the more important effect of increasing $\rho$. Here we present those details, considering each of $k, \lambda$ and $\rho$ in turn. First we consider $k$.

$\rho=0, \lambda \rightarrow \infty, k>0$

When parasitoid density is not extreme $(k \neq 0)$ but there continues to be no owner advantage $(\rho=0)$ and size advantage remains perfectly decisive $(\lambda \rightarrow \infty)$, for sufficiently large $\alpha$ there still exists an interior equilibrium $\left(p_{*}, p_{*}\right)$ that allows strategy $D$ to persist either statically or dynamically, although its strength is lower than for $k=0$. This result is illustrated by Figure $4(\mathrm{~b})$, where $k$ has increased from 0 to $5 \times 10^{-7}$ but all other parameter values are unchanged from $\S 3$. It may be surprising that a small increase in $k$ induces so marked a reduction in the amplitude of the limit cycle, but the larger and unsurprising point that is that increasing $k$ reduces the strength of strategy $D$ in the polymorphism (from 0.245 to 0.23 ): intuitively, size advantage in a contest is less likely to be favoured if a contest is less likely in the first place. When the value of $k$ is further increased, the strength of $D$ decreases further and the amplitude of the limit cycle continues to shrink around the unstable focus until it eventually morphs into a stable focus at $k \approx 7.03 \times 10^{-5}$ (with $p_{*} \approx 0.223$ ). Although $\left(p_{*}, p_{*}\right)$ remains a stable focus until $k \approx 0.206$, at which it morphs back into an unstable focus surrounded by a small-amplitude limit cycle, it remains the global attractor only until $k \approx 0.142$, at which value $(1,0)$ morphs from a saddle point into a second local attractor, this time a stable node. As $k$ increases from $k \approx 0.142$ to $k=0.25, D$ persists only if $\left(x_{1}(0), x_{2}(0)\right)$ lies in a steadily shrinking region 
between $(0,0)$ and a separatrix that joins a base-edge saddle point to a side-edge saddle point; and at $k \approx 0.25,\left(p_{*}, p_{*}\right)$ disappears entirely by merging with the saddle point at $(0,0)$ as $(1,0)$ becomes the global attractor. Thus, in this instance, strategy $D$ is bound to persist only if the probability that a host is never found does not exceed about $14 \%$; and if it exceeds $25 \%$, then it is guaranteed to be extinguished instead.

Although the effect of increasing $k$ is similar for other (sufficiently large) values of $\alpha$, the complexity of changes to phase-plane topology we have just described is not typical, being a consequence of $(\alpha, \theta)$ lying in region $E$ of Figure 5 ; for $(\alpha, \theta)$ in region $D$, the progression is far simpler. Suppose, for example, that $\alpha=0.6=\theta$, corresponding to Figure 3(a). Then as $k$ is steadily increased from $0,\left(p_{*}, p_{*}\right)$ remains a stable node as it moves steadily along $\Lambda$ towards the origin, merging with the saddle point there at $k=$ $\frac{37}{113} \approx 0.327$ as a stable node emerges from $(0,0)$ to progress along the base edge of $\Delta$ as $k$ increases further. This base-edge equilibrium remains the new global attractor until $k \approx 0.392$, at which it reaches $(1,0)$. Thus, in this instance, strategy $D$ is bound to persist as long as the probability that a host is never found does not exceed about $33 \%$; if it is larger than that, but less than about 39\%, then a polymorphism of $C$ and $U$ takes over; and if it exceeds that higher value, then only $C$ persists. An analytical expression for the critical value $k_{c}$ at which strategy $D$ disappears is $k_{c}=-\sigma_{0 b}(\alpha, \theta) /\{1-\theta+(2-\alpha) \alpha \theta\}$, where $\sigma_{0 b}$ is defined by (D.3); note that $k_{c}$ must be positive, because $\sigma_{0 b}<0$ for all $(\alpha, \theta)$ lying in region $\mathrm{D} \cup \mathrm{E}$ of Figure 5 (Appendix D). Note also that $k_{c}$ increases with $\alpha$.

The essence of the above discussion is that increasing $k$ decreases the strength of strategy $D$ by shifting $\left(p_{*}, p_{*}\right)$ along $\Lambda$ until it becomes unstable, ultimately to be absorbed by the origin. Note, however, that this description presumes $\rho=0$. When $\rho>0$, as discussed in $\S 4$, increasing $k$ still shifts $\left(p_{*}, p_{*}\right)$ towards the origin, but it it is no longer constrained to lie in $\Lambda$. For an illustration of this point, compare Figure 6(b) to Figure 6(d).

$\rho=0, \lambda<\infty, k=0$

Now we consider $\lambda$. When $\rho=0=k$ but $\lambda$ is finite, in place of (D.14) we obtain

$$
\begin{aligned}
2(1+\lambda)(1-\theta+\alpha \theta) \phi_{1}(p, p) & =-\{p+\theta(1-2 p)\} R(p) \\
\left.2(1+\lambda)(1-\theta+\alpha \theta) \phi_{2}(p, p)\right) & =\{(1-\theta)(1-p)+\theta p\} R(p)
\end{aligned}
$$

where

$$
\begin{aligned}
R(p) & =\left(4 \alpha-(1+\lambda)\left\{3 \alpha+\sigma_{0 b}\right\}\right) p^{2}+\alpha(1-\lambda) p-\lambda \sigma_{0 b}+(1-\alpha)^{2} \theta-1 \\
& =\left(\alpha-\sigma_{0 b}\right) p^{2}+\alpha p+(1-\alpha)^{2} \theta-1-\lambda Q(p)
\end{aligned}
$$

with $Q$ and $\sigma_{0 b}$ defined by (D.13) and (D.8), respectively. For $(p, p) \in \Lambda$ to be an interior equilibrium, we must have $\phi_{1}(p, p)=0=\phi_{2}(p, p)$, implying $R(p)=0$. Because $R\left(\frac{1}{2}\right)=$ 
$-\frac{5}{4}(1+\lambda)(1-\alpha)(1-\theta+\alpha \theta)$ and $\partial R /\left.\partial p\right|_{p=0}=\alpha(1-\lambda)$ are both negative, a zero in $\left(0, \frac{1}{2}\right)$ requires a large enough $\lambda$ to ensure $P(0)>0$, implying in particular that $\sigma_{0 b}<0$. So $(\alpha, \theta)$ must lie in region I $\cup$ II of Figure 1(b), and the interior equilibrium is absorbed by the origin when $P(0)=0$ or

$$
\lambda=\frac{(1-\alpha)^{2} \theta-1}{\sigma_{0 b}} .
$$

As long as $\lambda$ exceeds this value, however, an interior equilibrium $\left(p_{*}, p_{*}\right) \in \Lambda$ will exist, where $p_{*}$ is the only zero of $Q\left(p\right.$ on $\left(0, \frac{1}{2}\right)$; an explicit expression for $p_{*}$ can be found, but it is too unwieldy to be useful. Note that $R(p)=0$ implies $Q(p)=0$ in the limit as $\lambda \rightarrow \infty$.

For illustration, again consider $\alpha=0.6=\theta$, corresponding to Figure 3(a). Here, as $\lambda$ is steadily reduced, $\left(p_{*}, p_{*}\right)$ remains a stable node as it moves steadily down the line $x_{1}=x_{2}$ towards the origin, and the strength of strategy $D$ in the associated polymorphism of all three strategies does not differ appreciably from its value in the limit as $\lambda \rightarrow \infty$ until $\lambda$ reaches double figures; for example, $p_{*}=0.282$ for $\lambda=99$ and $p_{*}=0.229$ for $\lambda=9$ (as compared to $p_{*}=0.287$ in the limit as $\left.\lambda \rightarrow \infty\right)$. The decrease of $p_{*}$ then accelerates, but $\left(p_{*}, p_{*}\right)$ remains the global attractor until at $\lambda=(2213+1140 \sqrt{19}) / 2137 \approx 3.36$ it morphs from a stable focus into an unstable one, as a saddle point and a stable node emerge together at $x_{1} \approx 0.214$ on the base edge of $\Delta$. As $\lambda$ is further reduced, $\left(p_{*}, p_{*}\right)$ continues down $x_{1}=x_{2}$ towards the origin as the saddle point moves to the left, while the stable node becomes the global attractor and moves to the right; both the saddle point and $\left(p_{*}, p_{*}\right)$ are absorbed by the origin at $k \approx 3.05$, in agreement with (E.3). The phase-plane topology resembles that of Figure 2(b), except that there is an extra saddle point on the roof edge of $\Delta$ and $(1,0)$ is an unstable node. Subsequently, the base-edge node remains the global attractor for all $\lambda>1$.

For a second illustration, again consider $\alpha=0.5, \theta=0.8$, corresponding to Figure 4(a). Here, as $\lambda$ is steadily reduced, $\left(p_{*}, p_{*}\right)$ remains an unstable node surrounded by a globally attracting limit cycle until $\lambda=7$, at which a saddle point and a stable node emerge together at $x_{1}=\frac{1}{2}$ on the base edge of $\Delta$. As $\lambda$ is further reduced, $\left(p_{*}, p_{*}\right)$ continues down $x_{1}=x_{2}$ towards the origin, morphing from an unstable focus into an unstable node at $\lambda \approx 5.19$, while the saddle point moves to the left and the stable node becomes the global attractor and moves to the right; both the saddle point and $\left(p_{*}, p_{*}\right)$ are absorbed by the origin at $\lambda=4$, in agreement with (E.3). Subsequently, the base-edge node remains the global attractor for all $\lambda>1$. First we consider $k$.

$\rho>0, \lambda \rightarrow \infty, k=0$

Finally we consider $\rho$. In $\S 4$ we made the point that although a side-edge equilibrium corresponding to $(\alpha, \theta)$ in region $\mathrm{v} \cup$ vi of Figure 1 is impossible if $\rho=0$, for $\rho=0$ 
sufficiently large $\rho$ may induce a side-edge global attractor. In this regard, it is instructive to consider the limit as $\rho \rightarrow 1$. From (15) we find that $\phi_{2}\left(0, x_{2}\right)=\left(1-x_{2}\right) Q_{2}\left(x_{2}\right)$, where in place of the expression in (D.7), obtained for $\rho=0$, the quadratic polynomial $Q_{2}$ is now defined by

$$
Q_{2}\left(x_{2}\right)=\alpha \omega_{N}\left(1-\omega_{L} \theta\right)\left(1-x_{2}\right)-(1-\theta)\left\{\omega_{L} \omega_{N}\left(1+x_{2}^{2}\right)+\left(\omega_{L}^{2}+\omega_{N}^{2}\right) x_{2}\right\}
$$

with $\omega_{L}$ and $\omega_{N}$ defined by (1). Since $Q_{2}(0)=\alpha(1-\theta) \omega_{N}>0$ and $Q_{2}(1)=\theta-1<0$, there must exist precisely one $x_{2 s} \in(0,1)$ such that $Q_{2}\left(x_{2 s}\right)=0$. So a side-edge equilibrium $\left(0, x_{2 s}\right)$ exists. Expressions for $x_{2 s}$ and hence $r_{1}\left(0, x_{2 s}\right), r_{2}\left(0, x_{2 s}\right)$ as explicit functions of $\alpha$ and $\theta$ are again too cumbersome for presentation. As in Appendix D, however, we can show that both eigenvalues are always negative inside $S$. So $\left(0, x_{2 s}\right)$ not only always exists in the limit as $\rho \rightarrow 1$, but also is always a stable node.

\section{Appendix F. Ownership asymmetry in the absence of size asymmetry}

Here we present some analytical results obtained for the limiting case in which $\rho>0$ but $\lambda=1$ and $k=0$, so that ownership asymmetry obtains at high parasitoid density without size asymmetry. With $\lambda=1,(9)-(12)$ and (B.1)-(B.3) now imply

$$
\begin{aligned}
& \frac{d x_{1}}{d t}=\frac{1}{2} x_{1}\left\{\theta\left(1-x_{1}-x_{2}\right)+x_{2}\right\} S\left(x_{1}, x_{2}\right) \\
& \frac{d x_{2}}{d t}=-\frac{1}{2} x_{1}\left\{\theta x_{1}+(1-\theta)\left(1-x_{2}\right)\right\} S\left(x_{1}, x_{2}\right)
\end{aligned}
$$

where

$$
S\left(x_{1}, x_{2}\right)=\alpha \rho\left(1-x_{1}\right)^{2}+(1-\rho)\left(1-x_{2}\right)^{2}+2 x_{2}-\alpha\left(1+x_{1}^{2}\right) .
$$

Because the terms in squiggly brackets in (F.1) are both positive inside $\Delta$, any stationary points other than the three vertices of $\Delta$ must lie on an arc of the hyperbola with equation $S=0$. This curve intersects the interior of $\Delta$ only if $2 \alpha+\rho>1$, and then always intersects $x_{2}=0$ to the right of the origin. Hence there are no interior or side-edge stationary points (other than the vertices) if $2 \alpha+\rho<1$, in which case, $(1,0)$ is the global attractor; whereas if $2 \alpha+\rho>1$, then every point within $\Delta$ on the curve $S=0$ is a stationary point. In this case, as $t \rightarrow \infty,\left(x_{1}(t), x_{2}(t)\right)$ will approach the curve from above or below according to whether $\left(x_{1}(0), x_{1}(0)\right)$ lies above or below the curve, since $\frac{d x_{1}}{d t}$ is positive or negative according to whether $\left(x_{1}, x_{2}\right)$ lies above or below the curve while the signs are reversed for $\frac{d x_{2}}{d t}$. Hence strategy $C$ invariably persists in the absence of size asymmetry, whereas both strategy $D$ and strategy $U$ risk elimination through random drift, since every point along $S=0$ is only metastable. Note that $\theta$ becomes irrelevant in the absence of size asymmetry. 


\section{Appendix G. Phase-plane analysis for $\theta=\frac{1}{2}$ with $k=0, \lambda \rightarrow \infty$}

Proceeding as in Appendix D and using the same methods, in place of (D.3)-(D.5) we obtain

$$
\begin{aligned}
& 4(1+\alpha) r_{1}(0,0)=c(\alpha, \rho), \quad r_{2}(0,0)=-r_{1}(0,0) \\
& 4(1+\alpha) r_{1}(0,1)=2-(1-\rho) \alpha^{2}+2 \alpha \rho, \quad 2 r_{2}(0,1)=2-\alpha(1-\rho) \\
& 4(1+\alpha) r_{1}(1,0)=2 \alpha(\alpha+\rho+1)-1+\rho, \quad 2 r_{2}(1,0)=2 \alpha+\rho-1
\end{aligned}
$$

where

$$
c(\alpha, \rho)=2 \alpha-(1-\rho)\left(1-\alpha^{2}\right)
$$

and $0<\alpha, \rho<1$; the curve $c=0$ forms the right-hand boundary of the unshaded region 2 in Figure 8. Inspection of (G.1) shows that $(0,0)$ is invariably a saddle point, $(0,1)$ is invariably an unstable node and $(1,0)$ is a stable node, an unstable node or a saddle point according to whether

$$
\alpha<\frac{1}{2}\left\{\sqrt{\rho^{2}+3}-\rho-1\right\},
$$

$\alpha>\frac{1}{2}(1-\rho)$ or $\alpha$ lies between these bounds. In particular, $(1,0)$ is an attractor when $(\alpha, \rho)$ lies in region 1 of Figure 8. It is also the global attractor for that region.

A "roof-edge" equilibrium $\left(x_{1 r}, x_{2 r}\right)$ with $\phi_{1}\left(x_{1 r}, x_{2 r}\right)=0=\phi_{2}\left(x_{1 r}, x_{2 r}\right)$ and $x_{1 r}+x_{2 r}=$ 1 exists whenever $\alpha>\frac{1}{2}(1-\rho)$; here $\phi_{1}$ and $\phi_{2}$ are defined by (16). When it exists (that is, when $(1,0)$ is an unstable node), this equilibrium is invariably a saddle point because $r_{1}\left(x_{1 r}, x_{2 r}\right) r_{2}\left(x_{1 r}, x_{2 r}\right)<0$ throughout the relevant region.

For equilibria on the base edge of $\Delta$ where $0<x_{1}<1, x_{2}=0$ or on the side edge where $x_{1}=0,0<x_{2}<1$, (D.6) continues to hold but with

$$
\begin{gathered}
4(1+\alpha)^{2} Q_{1}\left(x_{1}\right)=\alpha\left\{\left\{\left(1+\alpha^{2}\right) \rho-(1+\alpha)^{2}\right\} x_{1}{ }^{2}\right. \\
\left.-\left\{2 \alpha^{2} \rho+(1+\alpha)(3 \rho-1)\right\} x_{1}\right\}-(1+\alpha) c(\alpha, \rho) \\
4(1+\alpha)^{2} Q_{2}\left(x_{2}\right)=-\left\{2 \alpha+(1-\rho)\left(1+\alpha^{2}\right)\right\} x_{2}{ }^{2} \\
-\{2 \rho+\alpha(1+\alpha)(1+3 \rho)\}+(1+\alpha) c(\alpha, \rho)
\end{gathered}
$$

in place of (D.7). A base-edge equilibrium occurs where $Q_{1}\left(x_{1}\right)=0$ for $x_{1} \in(0,1)$. Because $\partial Q_{1} /\left.\partial x_{1}\right|_{x_{1}=1}$ is invariably negative, no such equilibrium is possible if $Q_{1}(0)$ and $Q_{1}(1)$ are either both positive or both negative with $\partial Q_{1} /\left.\partial x_{1}\right|_{x_{1}=0}<0$. These constraints alone exclude much of the parameter square $\tilde{S}$ in Figure 8; and much of what remains is excluded by the constraint that if $Q_{1}(0)$ and $Q_{1}(1)$ are both negative with $\partial Q_{1} /\left.\partial x_{1}\right|_{x_{1}=0}>0$, then the equilibrium exists only if $Q_{1}\left(x_{1}\right)$ has a positive maximum, that is, only if $(\alpha, \rho)$ lies to the left of the curve with equation

$$
\alpha\left\{2 \alpha^{2} \rho+(1+\alpha)(3 \rho-1)\right\}^{2}=4(1+\alpha) c(\alpha, \rho)\left\{(1+\alpha)^{2}-\left(1+\alpha^{2}\right) \rho\right\},
$$


in which case there exists a pair of base-edge equilibria, $\left(x_{1 b}^{ \pm}, 0\right)$. Thus a base edgeequilibrium $\left(x_{1 b}^{+}, 0\right)$ exists when $(\alpha, \rho)$ lies in either the unshaded region 2 of Figure 8 or in the tiny region 5 , where a second base-edge equilibrium $\left(x_{1 b}^{-}, 0\right)$ also exists. Because $r_{1}\left(x_{1 b}^{+}, 0\right) r_{2}\left(x_{1 b}^{+}, 0\right)$ has a minimum of 0 while $r_{1}\left(x_{1 b}^{+}, 0\right)+r_{2}\left(x_{1 b}^{+}, 0\right)$ has a maximum of 0 , $\left(x_{1 b}^{+}, 0\right)$ is always a stable node; it is also the global attractor in region 2 . It continues to be a stable node in region 5 and typically remains the final destination of $\left(x_{1}(t), x_{2}(t)\right)$, but in this region there also exists either a stable side-edge node or a stable interior focus (according to whether region 5 intersects region 3 or region 4 ); whereas $r_{1}\left(x_{1 b}^{-}, 0\right)$ is always a saddle point.

A side-edge equilibrium $\left(0, x_{2}\right)=\left(0, x_{2 s}\right)$ occurs where $Q_{2}\left(x_{2}\right)=0$ for $x_{2} \in(0,1)$. Because $\partial Q_{2} /\left.\partial x_{2}\right|_{x_{2}=0}, \partial Q_{2} /\left.\partial x_{2}\right|_{x_{2}=1}$ and $Q_{2}(1)$ are all invariably negative, the condition for such an equilibrium to occur is $Q_{2}(0)>0$ or $c(\alpha, \rho)>0$. Thus a side-edge equilibrium $\left(0, x_{2 s}\right)$ occurs when $(\alpha, \rho)$ lies in either region 3 or region 4 of Figure 8; however, it is a stable node only when $(\alpha, \rho)$ lies in region 3 , since it is only here that $r_{1}\left(0, x_{1 s}\right) r_{2}\left(0, x_{1 s}\right)$ has a minimum of 0 while $r_{1}\left(0, x_{1 s}\right)+r_{2}\left(0, x_{1 s}\right)$ has a maximum of 0 . Except where region 3 intersects region $5,\left(0, x_{2 s}\right)$ is also the global attractor for region 3 .

For $(\alpha, \rho)$ in Region 4 of $\tilde{S}$ there always exists an interior equilibrium $\left(p_{*}, q_{*}\right) \in \Delta$ such that $\phi_{1}\left(p_{*}, q_{*}\right)=0=\phi_{2}\left(p_{*}, q_{*}\right)$. Its boundary is the curve with equation $d=0$, where

$$
d(\alpha, \rho)=\phi_{1}\left(0, x_{2 s}\right)
$$

is an extremely cumbersome expression that we do not present. This boundary extends from $\left(\alpha_{d}, 0\right)=(\sqrt{2}-1,0) \approx(0.414,0)$ to $\left(1, \rho_{d}\right) \approx(1,0.52)$ in Figure 8 , and it divides region 5 into a left-hand part that intersects with region 3 and a right-hand part that intersects with region 4 . In both parts, there are two local attractors. In the left-hand part, these attractors are both stable nodes, one on the side edge and one on the base edge of $\Delta$; in the right-hand part, the attractors are a stable base-edge node and a stable interior focus; and in both parts of region 5 there is a second base-edge equilibrium, a saddle point, which attracts a separatrix emanating from the unstable node at $(0,1)$, as in Figure $3(b)$. In both cases, the equilibrium to the left of this separatrix (either the side-edge node or the interior focus) has only a very small basin of attraction, and unless $\left(x_{1}(0), x_{2}(0)\right)$ lies within this tiny region, $\left(x_{1}(\infty), x_{2}(\infty)\right)$ is the base-edge node to the right of the separatrix. To illustrate, we pick a point in each part, each represented by a dot in Figure 8. For $(\alpha, \rho)=(0.39,0.1)$, the attractors are nodes at $\left(0.2472 \times 10^{-1}, 0\right)$ and $(0.4442,0)$, with the separatix ending at $\left(0.7459 \times 10^{-1}, 0\right)$; and for $(\alpha, \rho)=(0.42,0.02)$, the attractors are a focus at $\left(0.2394 \times 10^{-1}, 0.4131 \times 10^{-1}\right)$ and a node at $(0.5681,0)$, with the separatix ending at $\left(0.9817 \times 10^{-1}, 0\right)$. 